\title{
Invariant subspaces for certain tuples of operators with applications to reproducing kernel correspondences
}

\author{
Baruch Solel \\ Department of Mathematics, Technion \\ 32000 Haifa, Israel \\ e-mail: mabaruch@technion.ac.il
}

\begin{abstract}
The techniques developed by Popescu, Muhly-Solel and Good for the study of algebras generated by weighted shifts are applied to generalize results of Sarkar and of Bhattacharjee-Eschmeier-Keshari-Sarkar concerning dilations and invariant subspaces for commuting tuples of operators. In that paper the authors prove Beurling-Lax-Halmos type results for commuting tuples $T=\left(T_{1}, \ldots, T_{d}\right)$ operators that are contractive and pure; that is $\Phi_{T}(I) \leq I$ and $\Phi_{T}^{n}(I) \searrow 0$ where

$$
\Phi_{T}(a)=\Sigma_{i} T_{i} a T_{i}^{*}
$$

Here we generalize some of their results to commuting tuples $T$ satisfying similar conditions but for

$$
\Phi_{T}(a)=\Sigma_{\alpha \in \mathbb{F}_{d}^{+}} x_{|\alpha|} T_{\alpha} a T_{\alpha}^{*}
$$

where $\left\{x_{k}\right\}$ is a sequence of non negative numbers satisfying some natural conditions (where $T_{\alpha}=T_{\alpha(1)} \cdots T_{\alpha(k)}$ for $k=|\alpha|$ ). In fact, we deal with a more general situation where each $x_{k}$ is replaced by a $d^{k} \times d^{k}$ matrix.

We also apply these results to subspaces of certain reproducing kernel correspondences $E_{K}$ (associated with maps-valued kernels $K$ ) that are invariant under the multipliers given by the coordinate functions.
\end{abstract}




\section{Introduction}

A famous theorem due to Beurling, Lax and Halmos (see [14, Corollary 3.26]) describes the shift-invariant subspaces of $H_{G}^{2}(\mathbb{D})$ (where $H^{2}(\mathbb{D})$ is the classical Hardy space, $G$ is a Hilbert space and $H_{G}^{2}(\mathbb{D})$ is the vector-valued Hardy space and is isomorphic to $H^{2}(\mathbb{D}) \otimes G$ ). The theorem states that every shift invariant subspace of $H_{G}^{2}(\mathbb{D})$ is given by a partially isometric image of a vector-valued Hardy space $H_{D}^{2}(\mathbb{D})$. In fact, this partial isometry can be given by an inner function on $\mathbb{D}$ with values in $B(D, G)$.

This result was proved to be very important and there are many generalizations of it to various contexts. I will mention here only the ones that are most relevant to the discussion here.

The Hardy space $H^{2}(\mathbb{D})$ is a reproducing kernel Hilbert space (RKHS) where the kernel is the Szegö kernel $K(z, w)=\frac{1}{1-z \bar{w}}=\sum_{k=0}^{\infty} z^{k} \overline{w^{k}}(z, w \in \mathbb{D})$ and it has the Nevanlinna-Pick interpolation property. In [6] McCullough and Trent extended the BLH theorem to a general reproducing kernel Hilbert space $H_{K}$ where $K$ has the Nevanlinna-Pick property, in place of the Hardy space. For the case of weighted Bergman spaces, Ball and Bolotnikov ([1], [2]) studied the invariant subspaces and showed that they are the image (by partially isometric multipliers) of vector valued Hardy spaces.

In [15] J. Sarkar proved a BLH-type theorem for subspaces that are invatiant for a pure contraction and applied it to shift-invariant subspaces of RKHSs with special property that he calls "analytic" and that ensures that the coordinate function is a contractive multiplier. The Hardy space and the Bergman space are examples of analytic RKHSs. It is proved in these cases that an invariant subspace is a partially isometric image of a vector-valued Hardy space. Since the weighted Bergman spaces are analytic RKHSs, these results extend the results of Ball-Bolotnikov.

In [16] J. Sarkar extends the results of [15] to invariant subspaces of a pure row contraction of commuting operators. The place of the Hardy space is now played by the Drury-Arveson space (i.e, the symmetric Fock space). In [3] the authors continued the study of such commuting tuples and discussed also uniqueness (of dilations and of the partially isometric multipliers), the wandering subspaces associated with invariant subspaces and $K$-inner functions.

Another BLH type theorem that is relevant to our analysis here is in Popescu's [12]. In particular, [12, Theorem 3.3], where he characterized the

invariant subspaces under the constrained weighted shifts associated with a 
noncommutative variety is closely related to Theorem 5.9 (3) below. One should note though that his varieties are more general than what we use here but his definition of weights is more restrictive (the matrices $\left\{X_{k}\right\},\left\{R_{k}\right\}$ and $\left\{Z_{k}\right\}$ that we define below are, in his analysis, assumed all to be diagonal).

A general BLH theorem for RKHS $H_{K}$ with the property that $K$ has a complete Nevanlinna-Pick factor $s$ (that is, $K / s$ is a positive kernel) was recently proved by Clouâtre, Hartz and Schillo ([4]). They proved that, for a Hilbert space $\mathcal{E}$, a subspace $\mathcal{S} \subseteq H_{K} \otimes \mathcal{E}$ is invariant under the multipliers of $H_{s}$ (which, under the condition of the positivity of $K / s$, are also multipliers of $H_{K}$ ) if and only if there is a Hilbert space $\mathcal{F}$ such that $\mathcal{S}$ is the image of $H_{s} \otimes \mathcal{F}$ by a partially isometric multiplier.

In this paper, we study invariant subspaces for a tuple of commuting operators satisfying certain inequality and are pure in some natural sense. In [16] Sarkar studied commuting tuples $T=\left(T_{1}, \ldots, T_{d}\right)$ that are contractive and pure; that is $\Phi_{T}(I):=\sum_{i=1}^{d} T_{i} T_{i}^{*} \leq I$ and $\Phi_{T}^{n}(I) \searrow 0$ where

$$
\Phi_{T}(a)=\Sigma_{i} T_{i} a T_{i}^{*}
$$

Here, we replace this condition by a much more general one. Namely we write

$$
\Phi_{T}(a)=\Sigma_{\alpha \in \mathbb{F}_{d}^{+}} x_{|\alpha|} T_{\alpha} a T_{\alpha}^{*}
$$

where $\left\{x_{k}\right\}$ is a sequence of non negative numbers satisfying some natural conditions (where $T_{\alpha}=T_{\alpha(1)} \cdots T_{\alpha(k)}$ for $k=|\alpha|$ ). Thus, the tuples we study are commuting and satisfy

$$
\Sigma_{\alpha \in \mathbb{F}_{d}^{+}} x_{|\alpha|} T_{\alpha} T_{\alpha}^{*} \leq I
$$

and $\Phi_{T}^{n}(I) \searrow 0$. In fact, we deal with a more general situation where each $x_{k}$ is replaced by a $d^{k} \times d^{k}$ matrix $X_{k}$.

In Theorem 4.2 we present the BLH theorem for such tuples. The place of $H^{2}(\mathcal{D})$ (in [15]) or the Drury-Arveson space (in [16]) is now played by the "weighted Drury-Arveson space" $\mathcal{F}(\mathcal{R})$ that is defined and studied in Section 3. Among other things, it is shown there that it is the RKHS for some kernel defined on a domain that we denote by $D(X, \mathbb{C})$ (defined in (6) ). Both the kernel and the domain are defined in terms of the sequence $\left\{X_{k}\right\}$. The motivation for these definitions comes from the study of weighted Hardy algebras in 9 . 
The reader does not have to be familiar with the analysis of the weighted Hardy algebras and their representations as studied in [9] and in [5] but the results of these papers provide some of the motivation for our analysis here.

In Section 5 we discuss reproducing kernel correspondences $E_{K}$ associated with a map-valued kernel $K$ and we apply Theorem 4.2 to the row of multipliers by the coordinate functions. This is done in Theorem 5.7 and Theorem 5.9. In the study of the reproducing kernel correspondences we use results of [7] and of [5]. Since, unfortunately, these results have not been published yet, we shall provide all the details necessary for our discussion here.

In order to apply Theorem 4.2 to the multipliers of the reproducing kernel correspondence $E_{K}$ given by the coordinate functions $M_{S_{i}}$, we need to show that $M_{S}:=\left(M_{S_{1}}, \ldots, M_{S_{d}}\right)$ satisfies the conditions $\Phi_{M_{S}}(I) \leq I$ and $\Phi_{M_{S}}^{n}(I) \searrow 0$. It turns out (Theorem 5.11) that this holds if and only if the kernel $K_{c}^{R}$ (defined at the beginning of Section 5) is a factor of $K$. Since $K_{c}^{R}$ was shown by Good (in [5]) to have the complete Nevanlinna-Pick property, this fits well with the results of Clouâtre-Hartz-Schillo ([4]) mentioned above.

Since we will consider here both correspondences (which are $C^{*}$-Hilbert modules) and Hilbert spaces and it is customary, in the theory of $C^{*}$-Hilbert modules, to have inner products that are linear in the second term, we will use this convention also for Hilbert spaces. In particular, when studying a reproducing kernel Hilbert space $H_{K}$ with kernel functions $k_{z}$, we shall write $\left\langle k_{z}, k_{w}\right\rangle=K(z, w)$.

Finally, I wish to acknowledge helpful discussions with J. Eschmeier and with J. Sarkar regarding the results of this paper.

\section{Preliminaries}

We fix $0<d<\infty$. The following definition was introduced in [9] for the more general case where the Hilbert space $\mathbb{C}^{d}$ was replaced by a $W^{*}$ correspondence.

Definition 2.1 A sequence $\left\{X_{k}\right\}_{k=1}^{\infty}$ of operators will be called admissible if it satisfies the following conditions:

1. $X_{k} \in B\left(\mathbb{C}^{d} \otimes \mathbb{C}^{d} \otimes \cdots \otimes \mathbb{C}^{d}\right)=B\left(\left(\mathbb{C}^{d}\right)^{\otimes k}\right)$.

2. $X_{1}$ is invertible and $X_{k} \geq 0$ for all $k$. 


\section{3. $\lim \sup \left\|X_{k}\right\|^{\frac{1}{k}}<\infty$.}

Write $\mathbb{F}_{d}^{+}$for the words $\alpha=\alpha(1) \alpha(2) \ldots \alpha(k)$ on $d$ generators (written $\{1, \ldots, d\})$. For such a word $|\alpha|=k$. Note that $X \in B\left(\left(\mathbb{C}^{d}\right)^{\otimes k}\right)$ can be written as a matrix $\left(x_{\alpha, \beta}\right)$ indexed by words $\alpha, \beta \in \mathbb{F}_{d}^{+}$of length $k$ and a sequence $\left\{X_{k}\right\}$ with $X_{k} \in B\left(\left(\mathbb{C}^{d}\right)^{\otimes k}\right)$ can be written as a matrix $\left(x_{\alpha, \beta}\right)_{\alpha, \beta \in \mathbb{F}_{d}^{+}}$.

Associated with an admissible sequence $\left\{X_{k}\right\}$ we have another sequence of operators denoted $\left\{R_{k}\right\}_{k=0}^{\infty}$ where $R_{0}=I \in B(\mathbb{C})$ and, for $k \geq 1$,

$$
R_{k}=\left(\Sigma_{l=1}^{k} \Sigma_{\alpha \in F(k, l)} \otimes_{i=1}^{l} X_{\alpha(i)}\right)^{\frac{1}{2}}
$$

where

$$
F(k, l)=\left\{\alpha:\{1, \ldots, l\} \rightarrow \mathbb{N}: \Sigma_{i=1}^{l} \alpha(i)=k\right\} .
$$

Note that each $R_{k}$ is positive and invertible. Here, also, we can write $\left\{R_{k}^{2}\right\}_{k=0}^{\infty}$ as a matrix $\left(r_{\alpha, \beta}^{2}\right)_{\alpha, \beta \in \mathbb{F}_{d}^{+} \cup\{\emptyset\},|\alpha|=|\beta|}$.

The relationship between the $X_{k}$ 's and the $R_{k}$ 's is also given by

$$
R_{k}^{2}=\Sigma_{l=1}^{k} X_{l} \otimes R_{k-l}^{2}, \quad k \geq 1
$$

by [9, (4.7)]. If $d=1$ (so that $X_{k}=x_{k}$ and $R_{k}=r_{k}$ are scalars) then we have

$$
\frac{1}{1-\Sigma_{k \geq 1} x_{k} z^{k}}=\Sigma_{k \geq 0} r_{k}^{2} z^{k}
$$

and

$$
1-\frac{1}{\Sigma_{k \geq 0} r_{k}^{2} z^{k}}=\Sigma_{k \geq 1} x_{k} z^{k} .
$$

Given a tuple $T=\left(T_{1}, \ldots, T_{d}\right)$ of operators in $B(H)$, it will be convenient to view $T$ as an operator from $H^{(d)}$ to $H$ and for $k \geq 1$, we write $T^{(k)}:=$ $\left(T_{\alpha}\right)_{\alpha \in \mathbb{F}_{d}^{+},|\alpha|=k}$ (viewed as a row of operators or, equivalently, as a bounded operator from $\left(\mathbb{C}^{d}\right)^{\otimes k} \otimes H$ to $H$.) One can also write

$$
T^{(k)}=T\left(I_{E} \otimes T\right)\left(I_{E^{\otimes 2}} \otimes T\right) \cdots\left(I_{E^{\otimes(k-1)}} \otimes T\right): E^{\otimes k} \otimes H \rightarrow H
$$

where $E=\mathbb{C}^{d}\left(\right.$ as $\left.T: \mathbb{C}^{d} \otimes H \rightarrow H\right)$.

Given a tuple $T=\left(T_{1}, \ldots, T_{d}\right)$ and an admissible sequence $X=\left\{X_{k}\right\}=$ $\left(x_{\alpha, \beta}\right)$ such that the sum $\Sigma_{k=1}^{\infty} T^{(k)}\left(X_{k} \otimes I_{H}\right) T^{(k) *}=\Sigma_{|\alpha|=|\beta|} T_{\alpha} x_{\alpha, \beta} T_{\beta}^{*}$ is $w^{*}$ convergent,

we write $\Phi_{T}: B(H) \rightarrow B(H)$ for the (completely positive) map

$$
\Phi_{T}(a)=\Sigma_{k=1}^{\infty} T^{(k)}\left(X_{k} \otimes a\right) T^{(k) *}=\Sigma_{|\alpha|=|\beta|} T_{\alpha} x_{\alpha, \beta} a T_{\beta}^{*}
$$


where $T_{\alpha}=T_{\alpha(1)} T_{\alpha(2)} \cdots T_{\alpha(k)}$ if $|\alpha|=k$.

Also, we write

$$
D(X, H)=\left\{T=\left(T_{1}, \ldots, T_{d}\right): T_{i} \in B(H),\left\|\Phi_{T}(I)\right\|<1\right\}
$$

and

$$
D_{c}(X, H)=\left\{T \in D(X, H): T_{i} T_{j}=T_{j} T_{i}, i, j \in\{1, \ldots, d\}\right\}
$$

(the commuting $d$-tuples in $D(X, H)$ ),

$$
\bar{D}_{c}(X, H)=\left\{T \in \bar{D}(X, H): T_{i} T_{j}=T_{j} T_{i}, i, j \in\{1, \ldots, d\}\right\}
$$

and

$$
\bar{D}_{c p}=\left\{T \in \bar{D}(X, H): T_{i} T_{j}=T_{j} T_{i}, i, j \in\{1, \ldots, d\}, \Phi_{T}^{m}(I) \searrow 0\right\}
$$

where a tuple satisfying $\Phi_{T}^{m}(I) \searrow 0$ is said to be pure.

Example 2.2 Suppose each $X_{k}$ is a scalar. That is, $X_{k}=x_{k} I_{\left(\mathbb{C}^{d}\right) \otimes k}$. Then $\Phi_{T}(a)=\Sigma_{|\alpha|=|\beta|} x_{|\alpha|} T_{\alpha} a T_{\alpha}^{*}$ and

$$
D(X, H)=\left\{T=\left(T_{1}, \ldots, T_{d}\right): T_{i} \in B(H), \Sigma_{|\alpha|=|\beta|} x_{|\alpha|} T_{\alpha} T_{\alpha}^{*}<1\right\} .
$$

In particular, if $x_{1}=1$ and $x_{k}=0$ for $k>1$, we get $\Phi_{T}(a)=\sum_{i=1}^{d} T_{i} a T_{i}^{*}$ and $D(X, H)=\left\{T=\left(T_{1}, \ldots, T_{d}\right): T_{i} \in B(H), \Sigma_{i} T_{i} T_{i}^{*}<1\right\}$

Example 2.3 In the following examples we fix $H=\mathbb{C}$, so we write $z=$ $\left(z_{1}, \ldots, z_{d}\right)$ in place of $T$ and $\Phi_{z}(1)=\Sigma_{|\alpha|=|\beta|} z_{\alpha} x_{\alpha, \beta} \overline{z_{\beta}}$.

(1) When $d=1, \Phi_{z}(1)=\Sigma x_{k}|z|^{2 k}$. Using (3), we see that $\Phi_{z}(1)<1$ if and only if the series $\Sigma_{k \geq 0} r_{k}^{2}|z|^{2 k}$ converges. Since this is a power series, the set $D(X, \mathbb{C})$ is a disc in $\mathbb{C}$ in this case.

(2) Now set $d=2, X_{1}$ is the diagonal matrix $\operatorname{diag}(1,2)$ and $X_{k}=0$ for $k>1$. Then $\Phi_{z}(1)=\left|z_{1}\right|^{2}+2\left|z_{2}\right|^{2}$ and $D(X, \mathbb{C})$ is a convex set whose restriction to $\mathbb{R}^{2}$ is an ellipse.

(3) Set $d=2, X_{1}=I_{2}, X_{2}$ is the diagonal matrix diag $(1,120,120,1)$ and $X_{k}=0$ for $k>2$. Then $\Phi_{z}(1)=\left|z_{1}\right|^{2}+\left|z_{2}\right|^{2}+\left|z_{1}\right|^{4}+\left|z_{2}\right|^{4}+240\left|z_{1} z_{2}\right|^{2}$. In this case $(0,1 / 2),(1 / 2,0) \in D(X, \mathbb{C})$ but $(1 / 4,1 / 4)$ is not in $D(X, \mathbb{C})$. Thus $D(X, \mathbb{C})$ is not convex. 
In what follows it will be convenient to keep writing $E$ for $\mathbb{C}^{d}$. We shall also write $\mathcal{F}(E)$ for the full Fock space $\mathcal{F}(E)=\mathcal{F}\left(\mathbb{C}^{d}\right)=\Sigma_{k=0}^{\infty}\left(\mathbb{C}^{d}\right)^{\otimes k}$.

The following discussion and lemma will be useful to determine whether a commuting tuple is pure.

For a tuple $T=\left(T_{1}, \ldots, T_{d}\right)$ of commuting operators in $B(H)$ we write $b(T)$ for the row

$$
b(T)=\left(T^{(k)}\left(X_{k}^{1 / 2} \otimes I_{H}\right)\right)_{k=1}^{\infty}
$$

and can view it as an operator from $\mathcal{F}(E) \otimes H$ to $H$. We get

$$
\Phi_{T}(a)=b(T)\left(I_{\mathcal{F}(E)} \otimes a\right) b(T)^{*}
$$

for $a \in B(H)$.

Now compute

$$
\begin{aligned}
& \Phi_{T}^{2}(a)=\Sigma_{k=1}^{\infty} T^{(k)}\left(X_{k} \otimes \Sigma_{l=1}^{\infty} T^{(l)}\left(X_{l} \otimes a\right) T^{(l) *}\right) T^{(k) *}= \\
& \Sigma_{k, l \geq 1} T^{(k)}\left(I_{E^{\otimes k}} \otimes T^{(l)}\right)\left(X_{k} \otimes X_{l} \otimes a\right)\left(I_{E^{\otimes k}} \otimes T^{(l)}\right)^{*} T^{(k) *}= \\
& \sum_{m=2}^{\infty} T^{(m)}\left(\Sigma_{k+l=m} X_{k} \otimes X_{l} \otimes a\right) T^{(m) *} .
\end{aligned}
$$

Continuing this way, we get

$$
\Phi_{T}^{n}(a)=\Sigma_{m=n}^{\infty} T^{(m)}\left(\Sigma_{k_{1}+\cdots+k_{n}=m}\left(X_{k_{1}} \otimes \cdots \otimes X_{k_{n}}\right) \otimes a\right) T^{(n) *} .
$$

Write $X(m, n)=\Sigma_{k_{1}+\cdots+k_{n}=m}\left(X_{k_{1}} \otimes \cdots \otimes X_{k_{n}}\right) \in B\left(E^{\otimes m}\right)$ and let $b(T)^{(n)}$ be the row

$$
b(T)^{(n)}=\left(T^{(k)}\left(X(k, n) \otimes I_{H}\right)\right)_{k=1}^{\infty}
$$

which can be viewed as an operator in $B(\mathcal{F}(E) \otimes H, H)$, to get

$$
\Phi_{T}^{n}(I)=b(T)^{(n)} b(T)^{(n) *} .
$$

Note that, if $\Phi_{T}(I) \leq I$, then, for every $n,\left\|\Phi_{T}^{n}(I)\right\| \leq 1$ and $\left\|b(T)^{(n) *}\right\| \leq 1$. Thus, we get the following.

Lemma 2.4 Suppose $\Phi_{T}(I) \leq I$. Then $T$ is pure (that is, $\Phi_{T}^{n}(I) \searrow 0$ ) if and only if $\left\|b(T)^{(n) *} h\right\| \rightarrow 0$ for every $h$ in a subset of $H$ that spans a dense subspace of $H$. 


\section{$3 \mathcal{F}(\mathcal{R})$ (weighted Drury-Arveson space)}

For $k \geq 1$, we write

$$
\mathcal{R}_{k}^{0}=R_{k}\left(\mathbb{C}^{d}\right)^{\circledR k} \subseteq\left(\mathbb{C}^{d}\right)^{\otimes k}
$$

(where $\left(\mathrm{S}\right.$ is the symmetric tensor product) and $\mathcal{R}_{0}^{0}=\mathbb{C}$. Also write

$$
\mathcal{F}(\mathcal{R}):=\oplus_{k=0}^{\infty} \mathcal{R}_{k}^{0} \subseteq \mathcal{F}\left(\mathbb{C}^{d}\right)
$$

Proposition 3.1 For $z, w \in D(X, \mathbb{C})$, write

$$
K^{R}(z, w)=\Sigma_{k=0}^{\infty} z^{(k)} R_{k}^{2} w^{(k) *} .
$$

Then $K^{R}$ is a well defined positive kernel on $D(X, \mathbb{C}) \times D(X, \mathbb{C})$ and the corresponding RKHS $H_{K^{R}}$ is isomorphic to $\mathcal{F}(\mathcal{R})$ via the map u that sends the kernel function $k_{w}^{R}$ to $\Sigma_{k=0}^{\infty} R_{k} w^{(k) *}$.

Proof. To show that $K^{R}(z, w)$ is well defined (that is, the series is convergent) for $z, w \in D(X, \mathbb{C})$, we note first that, for $z \in D(X, \mathbb{C}), \Phi_{z}:=$ $\Sigma_{l=1}^{\infty} z^{(l)} X_{l} z^{(l) *}<1$ and, thus, $\Sigma_{l} \Phi_{z}^{l}$ converges.

Now note that, for $l, m$ we have $z^{(l+m)}=z^{(l)}\left(I_{\left(\mathbb{C}^{d}\right)^{(l)}} \otimes z^{(m)}\right)$ and, thus, $z^{(l+m)}\left(X_{l} \otimes X_{m}\right) z^{(l+m) *}=z^{(l)}\left(I_{\left(\mathbb{C}^{d}\right)^{(l)}} \otimes z^{(m)}\right)\left(X_{l} \otimes X_{m}\right)\left(I_{\left(\mathbb{C}^{d}\right)^{(l)}} \otimes z^{(m) *}\right) z^{(l) *}=$ $z^{(l)}\left(X_{l} \otimes z^{(m)} X_{m} z^{(m) *}\right) z^{(l) *}$. It follows that

$$
\Phi_{z}^{2}=\Sigma_{l, m}^{\infty} z^{(l+m)}\left(X_{l} \otimes X_{m}\right) z^{(l+m) *}=\Sigma_{k=1}^{\infty} z^{(k)}\left(\Sigma_{l+m=k} X_{l} \otimes X_{m}\right) z^{(k) *} .
$$

Similarly, for $k \geq 1$,

$$
\Phi_{z}^{l}=\Sigma_{k=1}^{\infty} z^{(k)}\left(\Sigma_{i_{1}+\cdots+i_{l}=k} X_{i_{1}} \otimes \cdots \otimes X_{i_{l}}\right) z^{(k) *}
$$

and, thus,

$$
\Sigma_{k=0}^{\infty} z^{(k)} R_{k}^{2} z^{(k) *}=\Sigma_{l} \Phi_{z}^{l}
$$

converges. Since this holds also for $w$, it follows that $K^{R}(z, w)$ is well defined. Positivity is clear and it is left to identify $H_{K^{R}}$ with $\mathcal{F}(\mathcal{R})$.

For this, note that $H_{K^{R}}$ is spanned by the functions

$$
k_{w}(z)=\Sigma_{k=0}^{\infty} z^{(k)}\left(R_{k}^{2} w^{(k) *}\right), \quad z \in D(X, \mathbb{C})
$$

where $w$ runs over $D(X, \mathbb{C})$. Now consider the linear map $u$ defined by

$$
u\left(k_{w}\right)=\sum_{k=0}^{\infty} R_{k} w^{(k) *} \in \mathcal{F}(\mathcal{R}) .
$$


We now need to show that $u$ preserves inner products. It will then follow that $u$ is well defined and can be extended to an isometry from $H_{R^{K}}$ to $\mathcal{F}(\mathcal{R})$. Since $H_{K^{R}}$ is generated by the functions $\left\{k_{w}: w \in D(X, \mathbb{C})\right\}$, it suffices to show that $u$ preserves inner products of these functions. So we compute, for $z, w \in D(X, \mathbb{C})$,

$$
\begin{aligned}
\left\langle k_{z}, k_{w}\right\rangle=K^{R}(z, w) & =\Sigma_{m=0}^{\infty} z^{(m)} R_{m}^{2} w^{(m) *}=\Sigma_{m}\left\langle R_{m} z^{(m) *} 1, R_{m} w^{(m) *} 1\right\rangle_{\left(\mathbb{C}^{d}\right)^{\otimes m}} \\
& =\Sigma_{m}\left\langle R_{m}\left(z^{*}\right)^{\otimes m}, R_{m}\left(w^{*}\right)^{\otimes m}\right\rangle_{\left(\mathbb{C}^{d}\right)^{\otimes m}}
\end{aligned}
$$

Thus, $u$ is a well defined isometry into $\mathcal{F}(\mathcal{R})$. To show that this map is surjective, note first that, for $\lambda \in \mathbb{T}$,

$$
u\left(k_{\bar{\lambda} w}\right)=\sum_{k=0}^{\infty} R_{k} \lambda^{k} w^{(k) *} \in \mathcal{F}(\mathcal{R}) .
$$

We now define, for every $\lambda \in \mathbb{T}, W_{\lambda} \in B(\mathcal{F}(\mathcal{R}))=B\left(\Sigma_{k} \oplus \mathcal{R}_{k}^{0}\right)$ by $W_{\lambda}=\Sigma_{k} \lambda^{k} Q_{k}$ (where $Q_{k}$ is the projection onto $\mathcal{R}_{k}^{0}$ ). Then $\left\{W_{\lambda}\right\}$ is a strongly continuous group of unitaries and (8) shows that the range of $u$ is invariant under each $W_{\lambda}$. Since, for every $k \geq 0, Q_{k}=\int W_{\lambda} \lambda^{-k} d \lambda$ (in the strong operator topology), we see that the range of $u$ (which is a closed subspace) is invariant under $Q_{k}$. Since $u\left(k_{w}\right)=\sum_{k=0}^{\infty} R_{k} w^{(k) *}$, we find that, for every $k \geq 0$ and every $w \in D(X, \mathbb{C}), R_{k} w^{(k) *}$ lies in the range of $u$. Thus, for every $k \geq 0, R_{k}\left(\mathbb{C}^{d}\right)^{\circledR k}$ is contained in the range of $u$. Since the range of $u$ is a closed subspace, $u$ is surjective.

Lemma 3.2 Given $T \in \bar{D}_{c}(X, H)$ and, for every $k \geq 1$, write $\mathcal{R}_{k}:=\mathcal{R}_{k}^{0} \otimes H$. Then

$$
\mathcal{R}_{k}=\left(\operatorname{span}\left\{\left(R_{k} \otimes A\right) T^{(k) *} h: A \in B(H), h \in H, T \in D_{c}(X, H)\right\}\right)^{-} .
$$

Proof. To prove that $\left(\operatorname{span}\left\{\left(R_{k} \otimes A\right) T^{(k) *} h: A \in B(H), h \in H, T \in\right.\right.$ $\left.\left.D_{c}(X, H)\right\}\right)^{-} \subseteq \mathcal{R}_{k}=R_{k}\left(\mathbb{C}^{d}\right)^{\circledR k} \otimes H$ it suffices to show that, for every $A \in B(H), h \in H$ and $T \in \bar{D}_{c}(X, H),(I \otimes A) T^{(k) *} h \in\left(\mathbb{C}^{d}\right)^{\circledR k} \otimes H$.

To prove it, we fix the following notation. $\left\{e_{i}\right\}_{i=1}^{d}$ is the standard orthonormal basis of $\mathbb{C}^{d}$, for every word $\alpha \in \mathbb{F}_{d}^{+}$of length $k$ we set $e_{\alpha}=e_{\alpha(1)} \otimes$ $\cdots \otimes e_{\alpha(k)} \in\left(\mathbb{C}^{d}\right)^{\otimes k}$ and, for a permutation $\pi \in S_{k}$ we write $\pi \alpha$ for the word obtained from $\alpha$ by applying $\pi$. Finally, we write $\tilde{e}_{\alpha}:=\Sigma_{\pi \in S_{k}} e_{\pi \alpha} \in\left(\mathbb{C}^{d}\right)^{\circledR k}$.

Using the fact that $T$ is a commuting tuple, it is easy to check that $(I \otimes A) T^{(k) *} h$ is a linear combination of $\tilde{e}_{\alpha} \otimes A T_{\alpha}^{*} h$ and, thus, is contained in $\left(\mathbb{C}^{d}\right)^{\Im k} \otimes H$. 
For the converse inclusion take $A=I$ and $T=\left(\lambda_{1} I, \lambda_{2} I, \ldots, \lambda_{d} I\right)$ (for $\lambda_{i} \in \mathbb{C}$ with $\left.\left|\lambda_{i}\right|<1\right)$. Then, for $h \in H, T^{*} h=\sum_{i=1}^{d} \overline{\lambda_{i}} e_{i} \otimes h$. Also,

$\left(T^{(2)}\right)^{*} h=\left(I_{\mathbb{C}^{d}} \otimes T^{*}\right)\left(\sum_{i=1}^{d} \overline{\lambda_{i}} e_{i} \otimes h\right)=\sum_{i=1}^{d} \overline{\lambda_{i}} e_{i} \otimes T^{*} h=\left(\sum_{i=1}^{d} \overline{\lambda_{i}} e_{i}\right) \otimes\left(\sum_{i=1}^{d} \overline{\lambda_{i}} e_{i}\right) \otimes h$.

Similarly,

$$
\left(T^{(k)}\right)^{*} h=\left(\sum_{i=1}^{d} \overline{\lambda_{i}} e_{i}\right)^{\otimes k} \otimes h .
$$

Since $\left(\mathbb{C}^{d}\right)^{\circledR k}$ is generated by vectors of the form $\left(\sum_{i=1}^{d} \overline{\lambda_{i}} e_{i}\right)^{\otimes k}$, we are done.

We now set $Z_{0}=I$ and, for $k>0$,

$$
Z_{k}=R_{k}^{-1}\left(I_{\mathbb{C}^{d}} \otimes R_{k-1}\right)
$$

(See [9, Equation (4.5)]).

On $\mathcal{F}\left(\mathbb{C}^{d}\right)$ we define, for every $1 \leq i \leq d$, the operator $\tilde{W}_{i}$ by

$$
\tilde{W}_{i} \theta=Z_{k+1}\left(e_{i} \otimes \theta\right)
$$

for $\theta \in\left(\mathbb{C}^{d}\right)^{\otimes k}$. So that $\tilde{W}_{i}$ is a weighted shift mapping $\left(\mathbb{C}^{d}\right)^{\otimes k}$ to $\left(\mathbb{C}^{d}\right)^{\otimes(k+1)}$.

We also define the tuple $W=\left\{W_{i}\right\}$ on $\mathcal{F}(\mathcal{R})$ by the compression of $\tilde{W}$ to $\mathcal{F}(\mathcal{R})$,

$$
W_{i}=P_{\mathcal{F}(\mathcal{R})} \tilde{W}_{i} \mid \mathcal{F}(\mathcal{R})
$$

Lemma 3.3 The sequence $\left\{Z_{k}\right\}$ is bounded (so that $\tilde{W}_{i}$ is a bounded operator for each i) and

$$
\tilde{W}_{i}^{*} \mathcal{F}(\mathcal{R}) \subseteq \mathcal{F}(\mathcal{R})
$$

Thus, $W_{i}^{*}=\tilde{W}_{i}^{*} \mid \mathcal{F}(\mathcal{R})$ and, in fact,

$$
W_{i}^{*} R_{k} w^{(k) *}=\tilde{W}_{i}^{*} R_{k} w^{(k) *}=R_{k} L_{i}^{*} w^{(k) *}
$$

for $w \in D(X, \mathbb{C})$ where $L_{i}:\left(\mathbb{C}^{d}\right)^{\otimes k} \rightarrow\left(\mathbb{C}^{d}\right)^{\otimes(k+1)}$ is the operator $L_{i} \theta=e_{i} \otimes \theta$. Also, for a word $\alpha, W_{\alpha}=P_{\mathcal{F}(\mathcal{R})} \tilde{W}_{\alpha} \mid \mathcal{F}(\mathcal{R})=P_{\mathcal{F}(\mathcal{R})} \tilde{W}_{\alpha}$ and $W_{\alpha}^{*}=\tilde{W}_{\alpha} \mid \mathcal{F}(\mathcal{R})$.

Proof. The boundedness of the sequence $\left\{Z_{k}\right\}$ follows from the proof of [9, Theorem 4.5]. For the other statement, write $L_{i}:\left(\mathbb{C}^{d}\right)^{\otimes k} \rightarrow\left(\mathbb{C}^{d}\right)^{\otimes(k+1)}$ for the operator $L_{i} \theta=e_{i} \otimes \theta$ and compute (using Equation (9) and the fact that, for every $\left.k, L_{i}^{*}\left(\mathbb{C}^{d}\right)^{(S(k+1)} \subseteq\left(\mathbb{C}^{d}\right)^{()(k)}\right)$

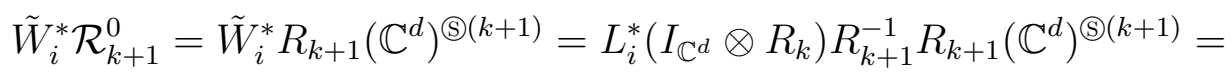




$$
L_{i}^{*}\left(I_{\mathbb{C}^{d}} \otimes R_{k}\right)\left(\mathbb{C}^{d}\right)^{\Im(k+1)}=R_{k} L_{i}^{*}\left(\mathbb{C}^{d}\right)^{((k+1)} \subseteq R_{k}\left(\mathbb{C}^{d}\right)^{\Im(k)}=\mathcal{R}_{k}^{0}
$$

It follows from the boundedness of $\left\{Z_{k}\right\}$, Equation (9) and the fact that $R_{k}$ is self adjoint, that

$$
I_{\mathbb{C}^{d}} \otimes R_{k-1}^{2}=\left(I_{\mathbb{C}^{d}} \otimes R_{k-1}\right)\left(I_{\mathbb{C}^{d}} \otimes R_{k-1}\right)^{*}=R_{k} Z_{k} Z_{k}^{*} R_{k} \leq C R_{k}^{2}
$$

where $C=\sup _{k}\left\|Z_{k}\right\|^{2}$.

Lemma 3.4 $W=\left\{W_{i}\right\} \in \bar{D}_{c p}(X, \mathcal{F}(\mathcal{R}))$.

Proof. It is shown in [9, (5.2)] that $\sum_{k=1}^{\infty} \tilde{W}^{(k)}\left(X_{k} \otimes I_{\mathcal{F}\left(\mathbb{C}^{d}\right)}\right) \tilde{W}^{(k) *}=I_{\mathcal{F}\left(\mathbb{C}^{d}\right)}-$ $P_{0}$ where $P_{0}$ is the projection onto $\mathbb{C} \subseteq \mathcal{F}\left(\mathbb{C}^{d}\right)$. Since $P_{\mathcal{F}(\mathcal{R})} \tilde{W}^{(k)}=W^{(k)}$, we have $\sum_{k=1}^{\infty} W^{(k)}\left(X_{k} \otimes I_{\mathcal{F}(\mathcal{R})}\right) W^{(k) *}=I_{\mathcal{F}(\mathcal{R})}-P_{0} \leq I$. Thus $W \in \bar{D}(X, \mathcal{F}(\mathcal{R}))$.

To show that $W$ is a commutative tuple recall first that, for $\xi \in\left(\mathbb{C}^{d}\right)^{\circledR(k+1)}$, $\tilde{W}_{i}^{*} R_{k+1} \xi=R_{k} L_{i}^{*} \xi$ (see the computation in the proof of Lemma 3.3). Thus, for such $\xi$ and $i, j$,

$$
W_{i}^{*} W_{j}^{*} R_{k+1} \xi=\tilde{W}_{i}^{*} \tilde{W}_{j}^{*} R_{k+1} \xi=\tilde{W}_{i}^{*} R_{k} L_{i}^{*} \xi=R_{k-1} L_{j}^{*} L_{i}^{*} \xi .
$$

Since $L_{i}^{*} L_{j}^{*}=L_{j}^{*} L_{i}^{*}$ on $\left(\mathbb{C}^{d}\right)^{(}(k+1), W_{i}^{*} W_{j}^{*}=W_{j}^{*} W_{i}^{*}$ for all $i, j$ implying that $W$ is a commutative tuple.

It is left to prove that it is pure. For this, we use Lemma 2.4. Since $\operatorname{span}\left\{R_{l} \xi^{\otimes l}: l \geq 0, \xi \in \mathbb{C}^{d}\right\}$ is dense in $\mathcal{F}(\mathcal{R})$, we need to show that

$$
b(W)^{(n) *} R_{l} \xi^{\otimes l} \rightarrow_{n \rightarrow \infty} 0 .
$$

Since, for $m>l, W^{(m) *} R_{l} \xi^{\otimes l}=0$, we have

$$
\begin{gathered}
b(W)^{(n) *} R_{l} \xi^{\otimes l}=\Sigma_{m=n}^{\infty} \oplus\left(X(m, n) \otimes I_{\mathcal{F}(\mathcal{R})}\right) W^{(m) *} R_{l} \xi^{\otimes l} \\
=\Sigma_{m=n}^{l} \oplus\left(X(m, n) \otimes I_{\mathcal{F}(\mathcal{R})}\right) W^{(m) *} R_{l} \xi^{\otimes l} \rightarrow 0
\end{gathered}
$$

as $n \rightarrow \infty$.

Consider the reproducing kernel Hilbert space $H_{K^{R}}$ as in Proposition 3.1. Recall that its elements are scalar-valued functions defined on $D(X, \mathbb{C})$ and a function $f: D(X, \mathbb{C}) \rightarrow \mathbb{C}$ is called a multiplier if for every $g \in H_{K^{R}}, f g$ also lies in $H_{K^{R}}$. In this case, we write $M_{f}$ for the operator that sends $g \in H_{K^{R}}$ to $f g$. By the closed graph theorem, $M_{f}$ is a bounded operator. 
Lemma 3.5 We keep the notation of Proposition 3.1.

(1) For every $1 \leq i \leq d$, write $z_{i}$ for the function on $D(X, \mathbb{C})$ defined by $z_{i}(w)=w_{i}$. Then $z_{i}$ is a multiplier of $H_{K^{R}}$ and we have

$$
W_{i} u=u M_{z_{i}}
$$

(2) $\cap \operatorname{Ker}\left(M_{z_{i}}^{*}-\bar{w}_{i}\right)=\mathbb{C} k_{w}^{R}$ for $w \in D(X, \mathbb{C})$.

Proof. To prove part (1) we first show that each $z_{n}$ is a multiplier. For this, we need to show that there is a positive constant $C$ such that the kernel $K^{\prime}(z, w):=C K^{R}(z, w)-z_{n} K^{R}(z, w) w_{n}^{*}$ is positive ([13, Theorem 6.28]). Note that, since $K^{R}(z, w)$ is a positive kernel, so is the kernel $K_{n}(z, w):=z_{n} K(z, w) w_{n}^{*}$ for every $n$. Thus, it will suffice to show that the kernel $K^{\prime \prime}(z, w):=C K^{R}(z, w)-\Sigma_{n} z_{n} K^{R}(z, w) w_{n}^{*}$ is positive for some $C$. So, we fix $w(1), \ldots, w(m)$ in $D(X, \mathbb{C})$ and consider the matrix $\left(K^{\prime \prime}(w(i), w(j))_{i, j}\right.$. The $i, j$ entry is

$K^{\prime \prime}(w(i), w(j))=\sum_{k=0}^{\infty} w(i)^{(k)} R_{k}^{2} w(j)^{(k) *}-\Sigma_{n} \Sigma_{k=0}^{\infty} w(i)_{n} w(i)^{(k)} R_{k}^{2} w(j)^{(k) *} \overline{w(j)_{n}}$.

Write $A(i, j)=\Sigma_{n} \Sigma_{k=0}^{\infty} w(i)_{n} w(i)^{(k)} R_{k}^{2} w(j)^{(k) *} \overline{w(j)_{n}}$. But then

$$
A(i, j)=\Sigma_{k=0}^{\infty} w(i)^{(k+1)}\left(I \otimes R_{k}^{2}\right) w(j)^{(k+1) *}
$$

and it follows from (12) that $A$ is smaller than $B$ where

$$
B(i, j)=C \Sigma_{k=0}^{\infty} w(i)^{(k+1)} R_{k+1}^{2} w(j)^{(k+1) *}
$$

for $C=\sup \left\|Z_{k}\right\|^{2}$. Thus, $K^{\prime \prime}$ is a positive kernel and each $z_{i}$ is a multiplier. It follows that $M_{z_{i}}^{*} k_{w}=\overline{w_{i}} k_{w}$.

To prove (13), we compute

$$
u M_{z_{i}}^{*} u^{*}\left(\Sigma_{k=0}^{\infty} R_{k} w^{(k) *}\right)=u M_{z_{i}}^{*} k_{w}^{R}=\overline{w_{i}} u\left(k_{w}^{R}\right)=\bar{w}_{i} \Sigma_{k=0}^{\infty} R_{k} w^{(k) *} .
$$

But, using (11) and the fact that $W_{i}^{*}$ vanishes on the 0 th term $(\mathbb{C})$, we get

$$
u M_{z_{i}}^{*} u^{*}\left(\sum_{k=0}^{\infty} R_{k} w^{(k) *}\right)=W_{i}^{*}\left(\sum_{k=0}^{\infty} R_{k} w^{(k) *}\right) .
$$

For part (2), note that $\cap K \operatorname{Ker}\left(M_{z_{i}}^{*}-\bar{w}_{i}\right) \supseteq \mathbb{C} k_{w}^{R}$ is true in general. For the other inclusion, assume $\Sigma R_{k} \xi_{k} \in \cap \operatorname{Ker}\left(W_{i}^{*}-\bar{w}_{i}\right)$ for $\xi_{k} \in\left(\mathbb{C}^{d}\right)^{\circledR k} \subseteq\left(\mathbb{C}^{d}\right)^{\otimes k}$. We compute

$\bar{w}_{i}\left(\Sigma R_{k} \xi_{k}\right)=W_{i}^{*}\left(\Sigma R_{k} \xi_{k}\right)=\tilde{W}_{i}^{*}\left(\Sigma R_{k} \xi_{k}\right)=\Sigma L_{i}^{*} Z_{k}^{*} R_{k} \xi_{k}=\Sigma L_{i}^{*}\left(I_{\mathbb{C}^{d}} \otimes R_{k-1}\right) \xi_{k}=$ 


$$
\Sigma R_{k-1} L_{i}^{*} \xi_{k}
$$

Thus, for every $k$ and every $i, \bar{w}_{i} R_{k} \xi_{k}=R_{k} L_{i}^{*} \xi_{k+1}$ and, since $R_{k}$ is invertible,

$$
\bar{w}_{i} \xi_{k}=L_{i}^{*} \xi_{k+1} \text {. }
$$

It is now easy to check that, setting $\xi_{0}=1$, there is only a unique solution (or use the fact that it is known for the Drury-Arveson space and what we did above is reducing it to this case) and this proves (2).

\section{Dilations and invariant subspaces}

For $T \in \bar{D}_{c p}(X, H)$, we write $\Delta_{*}(T)=\left(I_{H}-\Phi_{T}(I)\right)^{\frac{1}{2}}$ and $\mathcal{D}_{*}:=\overline{\Delta_{*}(T)(H)}$. We also define the operator

$$
\Pi(T): H \rightarrow \mathcal{F}(\mathcal{R}) \otimes \mathcal{D}_{*}
$$

by

$$
\Pi(T)=\left(I_{\mathcal{F}(\mathcal{R})} \otimes \Delta_{*}(T)\right)\left(I_{H} \quad\left(R_{1} \otimes I_{H}\right) T^{*}\left(R_{2} \otimes I_{H}\right)\left(T^{(2)}\right)^{*} \ldots\right)^{T} .
$$

That is,

$$
\Pi(T) h=\left(I_{\mathcal{F}(\mathcal{R})} \otimes \Delta_{*}(T)\right)\left(h \oplus\left(R_{1} \otimes I_{H}\right) T^{*} h \oplus\left(R_{2} \otimes I_{H}\right)\left(T^{(2)}\right)^{*} h \oplus \ldots\right)
$$

for $h \in H$. (See [9, Definition 5.3], [10] and [11] where this map is referred to as the Poisson kernel). It generalizes the operator $\Pi_{c}$ defined in [3] which is shown there to be a dilation of a commuting tuple to a Hilbert space valued Drury-Arveson space. Indeed, if $R_{k}=I$ for all $k, \mathcal{F}(\mathcal{R})$ is the Drury-Arveson space as was shown in Lemma 1 .

Lemma 4.1 For every $T \in \bar{D}_{c p}(X, H)$, the map $\Pi(T): H \rightarrow \mathcal{F}(\mathcal{R}) \otimes \mathcal{D}_{*}$ is an isometry and satisfies,

$$
\Pi(T) T_{i}^{*}=\left(W_{i}^{*} \otimes I_{\mathcal{D}_{*}}\right) \Pi(T)
$$

for every $1 \leq i \leq d$.

Proof. The proof that $\Pi(T)$ is an isometry is the same as in [9, Lemma 5.4] (using the assumption that $T$ is pure) and the rest follows from the proof of [9, Lemma 5.5 1.]. To see this, replace $\mathfrak{z}$ there by $T, K(\mathfrak{z})$ there by $\Pi(T)$ here and $\xi$ there by $e_{i}$ here.

The following result generalizes [3, Theorem 4.1]. 
Theorem 4.2 Let $T \in \bar{D}_{c p}(X, H)$ and $\mathcal{S} \subseteq H$ be a subspace of $H$. Then $\mathcal{S}$ is a joint T-invariant subspace if and only if there exists a Hilbert space $\mathcal{D}$ and a partial isometry $\Pi \in B(\mathcal{F}(\mathcal{R}) \otimes \mathcal{D}, H)$ with $T_{i} \Pi=\Pi\left(W_{i} \otimes I_{\mathcal{D}}\right)$ for all $1 \leq i \leq d$ and

$$
\mathcal{S}=\Pi(\mathcal{F}(\mathcal{R}) \otimes \mathcal{D}) .
$$

Proof. It is clear that if $\mathcal{S}=\Pi(\mathcal{F}(\mathcal{R}) \otimes \mathcal{D})$ and $T_{i} \Pi=\Pi\left(W_{i} \otimes I_{\mathcal{D}}\right)$, then $\mathcal{S}$ is a joint $T$-invariant subsplace of $H$.

For the other direction we consider the restriction $T \mid \mathcal{S} \in B(\mathcal{S})$. Write $\iota_{\mathcal{S}}: \mathcal{S} \rightarrow H$ for the inclusion map and $P_{\mathcal{S}}$ for its adjoint, the (orthogonal) projection onto $\mathcal{S}$. It is easy to check that, for every $k \geq 1,(T \mid \mathcal{S})^{(k)}=$ $T^{(k)} \mid E^{\otimes k} \otimes \mathcal{S}$ and, thus,

$$
\Phi_{T \mid \mathcal{S}}(a)=\sum_{k=1}^{\infty} T^{(k)}\left(X_{k} \otimes P_{\mathcal{S}} a P_{\mathcal{S}}\right) T^{(k) *} .
$$

It follows that $T \in \bar{D}_{c}(X, H)$ and, to apply Lemma 4.1 to $T \mid \mathcal{S}$, we need to check that it is also pure. Now

$$
\begin{aligned}
\Phi_{T \mid \mathcal{S}}^{2}(a)= & \Sigma_{k=1}^{\infty} T^{(k)}\left(X_{k} \otimes P_{\mathcal{S}} \Sigma_{m=1}^{\infty} T^{(m)}\left(X_{m} \otimes P_{\mathcal{S}} a P_{\mathcal{S}}\right) T^{(m) *} P_{\mathcal{S}}\right) T^{(k) *}= \\
& \Sigma_{k, m} T^{(k)}\left(X_{k} \otimes P_{\mathcal{S}} T^{(m)}\left(X_{m} \otimes P_{\mathcal{S}} a P_{\mathcal{S}}\right) T^{(m) *} P_{\mathcal{S}}\right) T^{(k) *}
\end{aligned}
$$

Since $\mathcal{S}$ is $T$-invariant, we have $P_{\mathcal{S}} T^{(m)}\left(I_{E^{\otimes m}} \otimes P_{\mathcal{S}}\right)=T^{(m)}\left(I_{E^{\otimes m}} \otimes P_{\mathcal{S}}\right)$ and, therefore,

$$
\begin{gathered}
\Phi_{T \mid \mathcal{S}}^{2}(a)=\Sigma_{k, m} T^{(k)}\left(X_{k} \otimes T^{(m)}\left(X_{m} \otimes P_{\mathcal{S}} a P_{\mathcal{S}}\right) T^{(m) *}\right) T^{(k) *}= \\
\Sigma_{k, m} T^{(k+m)}\left(X_{k} \otimes X_{m} \otimes P_{\mathcal{S}} a P_{\mathcal{S}}\right) T^{(k+m) *} .
\end{gathered}
$$

The same computation for $T$ (instead of $T \mid \mathcal{S}$ ) yields

$$
\Phi_{T}^{2}(a)=\Sigma_{k, m} T^{(k+m)}\left(X_{k} \otimes X_{m} \otimes a\right) T^{(k+m) *}
$$

and, thus

$$
\Phi_{T \mid \mathcal{S}}^{2}(a)=\Phi_{T}^{2}\left(P_{\mathcal{S}} a P_{\mathcal{S}}\right) .
$$

Continuing in this way (see also the computation in [9, Proof of Theorem 4.5]), we get, fot every $n \geq 1$,

$$
\Phi_{T \mid \mathcal{S}}^{n}(a)=\Phi_{T}^{n}\left(P_{\mathcal{S}} a P_{\mathcal{S}}\right)
$$

and, since $T$ is pure, so is $T \mid \mathcal{S}$. 
The rest of the argument proceeds as in [3, Proof of Theorem 4.1]. We use Lemma 4.1 to get an isometry $\Pi_{\mathcal{S}}: \mathcal{S} \rightarrow \mathcal{F}(\mathcal{R}) \otimes \mathcal{D}$ that satisfies $\Pi_{\mathcal{S}} P_{\mathcal{S}} T_{i}^{*}=$ $\left(W_{i}^{*} \otimes I_{\mathcal{D}}\right) \Pi_{\mathcal{S}}$. Finally, let

$$
\Pi:=\iota_{\mathcal{S}} \circ \Pi_{\mathcal{S}}^{*}: \mathcal{F}(\mathcal{R}) \otimes \mathcal{D} \rightarrow H
$$

be the required map.

\section{$5 \quad$ Invariant subspaces in reproducing kernel correspondences}

With the notation set up above, we now consider the kernel

$$
K^{R}(V, W)(a)=\Sigma_{k=0}^{\infty} V^{(k)}\left(R_{k}^{2} \otimes a\right) W^{(k) *}=\Sigma_{|\alpha|=|\beta|} V_{\alpha} r_{\alpha, \beta}^{2} a W_{\beta}^{*}
$$

where $V, W \in D(X, H)$ and $a \in B(H)$. This defines a completely positive maps-valued kernel on $D(X, H) \times D(X, H)$ with values in the bounded maps on $B(H)$. In [9, Theorem 4.5] it was shown that it is well defined (where the sum converges in the norm topology) and in [5] J. Good studied this kernel (in the more general context of $W^{*}$-correspondence $E$ over a von Neumann algebra $M$ ) and the reproducing kernel $W^{*}$-correspondence associated to this kernel. She denoted it $\mathcal{H}^{2}(X, \sigma)$ (where $\sigma$ is the representation of $M$ on $H$ ).

Since, here, we are interested in the commuting tuples we write $K_{c}^{R}(V, W)$ for the restriction of $K^{R}$ to $D_{c}(X, H) \times D_{c}(X, H)$.

Now we consider a kernel

$$
K: D_{c}(X, H) \times D_{c}(X, H) \rightarrow B_{*}(B(H), B(H))
$$

that is completely positive. That means that, given $\left\{V_{i}\right\}_{i=1}^{n}$ with $V_{i} \in$ $D_{c}(X, H)$, the map

$$
\left(a_{i, j}\right) \mapsto\left(K\left(V_{i}, V_{j}\right)\left(a_{i, j}\right)\right)
$$

on $M_{n}(B(H))$ is completely positive.

Associated with such a kernel one defines a $W^{*}$-correspondence $E_{K}$ over $B(H)$. The details of the following statements can be found in [7, Chapter 3] or in [5].

In general, map-valued cp kernels are functions $K: \Sigma \times \Sigma \rightarrow B_{*}(N, L)$ where $\Sigma$ is a set and $N, L$ are $W^{*}$-algebras and it is completely positive 
in the sense that, given $n$ and points $z_{1}, \ldots, z_{n}$ in $\Sigma$, the matrix (of maps) $\left(K\left(z_{i}, z_{j}\right)\right)_{i, j=1}^{n}$ represents a (normal) completely positive map from $M_{n}(N)$ to $M_{n}(L)$. Associated with such a kernel one gets an $N-L W^{*}$-correspondence $E_{K}$. The elements of $E_{K}$ are functions $f: \Sigma \rightarrow B(N, L)$.

Conversely, given such a correspondence, with reproducing property as below, one gets a completely positive maps-valued kernel.

Although the details can be found in [7] and in [5], we sketch those details about the construction of $E_{K}$ that we shall need. Assume a map-valued cp kernel $K: \Sigma \times \Sigma \rightarrow B_{*}(N, L)$ is given. We define functions $k_{(a, w)}: \Sigma \rightarrow$ $B_{*}(N, L)$, for $a \in N$ and $w \in \Sigma$ by

$$
k_{(a, w)}(z)(b)=K(z, w)\left(b a^{*}\right)
$$

for $z \in \Sigma$ and $b \in N$. These functions generate $E_{K}$ as an $N-L$ correspondence where the left action of $N$ on these functions is defined by

$$
d \cdot k_{(a, w)}=k_{\left(a d^{*}, w\right)}
$$

and the $L$-valued inner product of these functions is given by

$$
\left\langle k_{(b, z)}, k_{(a, w)}\right\rangle=k_{(a, w)}(z)(b)=K(z, w)\left(b a^{*}\right) .
$$

Thus, the elements of $E_{K}$ are functions $f: \Sigma \rightarrow B_{*}(N, L)$ and $E_{K}$ is generated, as a $W^{*}$-module by $\left\{k_{(a, w)} \cdot c: a \in N, w \in \Sigma, c \in L\right\}$. The left action of $N$ on $f \in E_{K}$ is defined by $(a \cdot f)(z)(b)=f(z)(b a)$.

For $f \in E_{K}$, we have

$$
f(z)(a)=\left\langle k_{(a, z)}, f\right\rangle
$$

so these are the kernel functions that induce point evaluations. We also have

$$
\left\langle k_{(b, z)} \cdot c, k_{(a, w)} \cdot d\right\rangle=c^{*} K(z, w)\left(b a^{*}\right) d \in L .
$$

The following definition and theorem can be found in [7, Definition 46, Lemma 47, Theorem 48].

Definition 5.1 Let $N$ and $L$ be $W^{*}$-algebras and let $E_{K}$ be a reproducing kernel $W^{*}$-correspondence from $N$ to $L$ with associated cp kernel $K: \Sigma \times \Sigma \rightarrow$ $B_{*}(N, L)$. Then a function $\phi: \Sigma \rightarrow L$ is called a multiplier of $E_{K}$ if for each $f \in E_{K}$, the function $\phi f$, defined by $(\phi f)(z)(b)=\phi(z) f(z)(b)$ is in $E_{K}$. 
Theorem 5.2 Let $N$ and $L$ be $W^{*}$-algebras and let $E_{K}$ be a reproducing kernel $W^{*}$-correspondence from $N$ to $L$ with associated cp kernel $K: \Sigma \times \Sigma \rightarrow$ $B_{*}(N, L)$. Then

(1) If $\phi$ is a multiplier of $E_{K}$ then the map $M_{\phi}: E_{K} \rightarrow E_{K}$, defined by $M_{\phi} f=\phi f$, is in $\mathcal{L}\left(E_{K}\right) \cap \varphi_{E_{K}}(N)^{\prime}$.

(2) If $\phi$ is a multiplier of $E_{K}$ then, for all $(a, w) \in N \times \Sigma, M_{\phi}^{*} k_{a, w}=$ $k_{a, w} \phi(w)^{*}$.

(3) A function $\phi: \Sigma \rightarrow L$ is a multiplier of $E_{K}$ with $\left\|M_{\phi}\right\| \leq 1$ if and only if the map $K_{\phi}: \Sigma \times \Sigma \rightarrow B_{*}(N, L)$ defined by $K_{\phi}(w, z)=(i d-$ $A d(\phi(w), \phi(z))) \circ K(w, z)$ is a cp kernel.

Assume from now on that $N=L=B(H), \Sigma=D_{c}(X, H)$ and $K$ is such a map-valued kernel (of maps on $B(H)$ ).

Definition 5.3 Suppose $K$ is as above and, for every $i$, the map $S_{i}: D_{c}(X, H) \rightarrow$ $B(H)$, defined by $S_{i}(T)=T_{i}$, is a bounded multiplier of $E_{K}$. If $S:=\left(S_{i}\right)$ satisfies

$$
\sum_{k=1}^{\infty} M_{S}^{(k)}\left(X_{k} \otimes I_{E_{K}}\right) M_{S}^{(k) *} \leq I
$$

We shall say that $E_{K}$ is an $(\mathrm{X}, \mathrm{H})$-contractive reproducing kernel correspondence.

Proposition 5.4 Suppose $\Phi_{M_{S} \otimes_{B(H)}}(I) \leq I$ then $\Phi_{M_{S} \otimes_{B(H)}}^{n}(I) \searrow 0$. Thus, if $E_{K}$ is an $(X, H)$-contractive reproducing kernel correspondence then

$$
M_{S} \otimes_{B(H)} I_{H} \in \bar{D}_{c p}\left(X, E_{K} \otimes_{B(H)} H\right) .
$$

Proof. For simplicity, we will write here $G$ for $E_{K} \otimes_{B(H)} H, M_{i}$ for $M_{S_{i}} \otimes H \in B(G)$ and $M=\left(M_{1}, \ldots, M_{d}\right)$ for $M_{S} \otimes I_{H}$. By Lemma 2.4, we need to show that $\left\|b(M)^{(n) *}\left(k_{a, T} \cdot c \otimes_{B(H)} h\right)\right\| \rightarrow_{n} 0$ for $a, c \in B(H)$, $T \in D_{c}(X, H)$ and $h \in H$ (where $k_{a, T}$ is the kernel function in $E_{K}$ and $b(M)$ is the row defined in (7) with $M$ in place of $T$ ).

Recall that $S_{i}$ is the $i$ th coordinate function on $D_{c}(X, H)$ and $M_{S_{i}}$ is the corresponding multiplier. Thus $M_{S_{i}}^{*} k_{a, T} \otimes_{B(H)} f=k_{a, T} \cdot T_{i}^{*} \otimes_{B(H)} f=$ $k_{a, T} \otimes_{B(H)} T_{i}^{*} f$. It follows that

$$
M_{S}^{*} k_{a, T} \otimes_{B(H)} c f=M_{S}^{*} k_{a, T} \cdot c \otimes_{B(H)} f=k_{a, T} \otimes_{B(H)} T^{*} c f .
$$


Continuing this way, we can write, for every $m$,

$$
M^{(m) *}\left(k_{a, T} \cdot c \otimes_{B(H)} h\right)=M_{S}^{(m) *} k_{a, T} \otimes_{B(H)} c f=k_{a, T} \otimes_{B(H)} T^{(m) *} c f
$$

and

$$
\begin{gathered}
b(M)^{(n) *}\left(k_{a, T} \cdot c \otimes_{B(H)} h\right)=\Sigma_{m=n}^{\infty} \oplus\left(X(m, n) \otimes I_{G}\right) M^{(m) *}\left(k_{a, T} \cdot c \otimes_{B(H)} h\right)= \\
k_{a, T} \otimes_{B(H)}\left(\Sigma_{m=n}^{\infty} \oplus\left(X(m, n) \otimes I_{G}\right) T^{(m) *} c h\right)=k_{a, T} \otimes_{B(H)} b(T)^{(n) *} c h .
\end{gathered}
$$

But $\left\|b(T)^{(n) *} \operatorname{ch}\right\|^{2}=\left\langle\Phi_{T}^{n}(I) c h, c h\right\rangle$ and, since $T \in D(X, H), \Phi_{T}^{n}(I) \searrow 0$ by [9. Lemma 5.4 (4)] and this completes the proof.

Proposition 5.5 (1) For every $1 \leq i \leq d, S_{i}$ is a multiplier of $E_{K_{c}^{R}}$.

(2) There is an isomorphism

$$
\Lambda: E_{K_{c}^{R}} \otimes_{B(H)} H \rightarrow \mathcal{F}(\mathcal{R}) \otimes H
$$

that satisfies

$a$.

$$
\Lambda^{*}\left(W_{i} \otimes I_{H}\right) \Lambda=M_{S_{i}} \otimes I_{H}
$$

for every $1 \leq i \leq d$.

b. For $b \in B(H)$,

$$
\Lambda\left(\varphi_{E_{K_{c}^{R}}}(b) \otimes I_{H}\right)=\left(I_{\mathcal{F}(\mathcal{R})} \otimes b\right) \Lambda
$$

where $\varphi_{E_{K_{c}^{R}}}$ is the left action of $B(H)$ on $E_{K_{c}^{R}}$.

(3) $E_{K_{c}^{R}}$ is an $(X, H)$-contractive reproducing kernel correspondence.

Proof. Given $T \in D_{c}(X, H)$ we have, for $k \geq 1$ and $1 \leq i \leq d$,

$$
T^{(k+1)} L_{i}=T_{i} T^{(k)}
$$

where $L_{i}\left(f_{1} \otimes \cdots \otimes f_{k} \otimes h\right)=e_{i} \otimes f_{1} \otimes \cdots \otimes f_{k} \otimes h\left(f_{j} \in E=\mathbb{C}^{d}, h \in H\right)$. To check this, just compute $T^{(k+1)} L_{i}\left(f_{1} \otimes \cdots \otimes f_{k} \otimes h\right)=T^{(k+1)}\left(e_{i} \otimes f_{1} \otimes \cdots \otimes\right.$ $\left.f_{k} \otimes h\right)=T\left(I_{E} \otimes T^{(k)}\right)\left(e_{i} \otimes f_{1} \otimes \cdots \otimes f_{k} \otimes h\right)=T\left(e_{i} \otimes T^{(k)}\left(f_{1} \otimes \cdots \otimes f_{k} \otimes h\right)\right)=$ $T_{i} T^{(k)}\left(f_{1} \otimes \cdots \otimes f_{k} \otimes h\right)$. 
To prove that $S_{i}$ is a multiplier of $E_{K_{c}^{R}}$, consider, for $T, V \in D_{c}(X, H)$ and $a, b \in B(H)$,

$$
\begin{gathered}
S_{i}(T) K_{c}^{R}(T, V)\left(a^{2}\right) S_{i}(V)^{*}=\sum_{k=0}^{\infty} T_{i} T^{(k)}\left(R_{k}^{2} \otimes b a^{*}\right) V^{(k) *} V_{i}^{*}= \\
\sum_{k=0}^{\infty} T^{(k+1)} L_{i}\left(R_{k}^{2} \otimes b a^{*}\right) L_{i}^{*} V^{(k+1) *} .
\end{gathered}
$$

Using (9) we have, for $f_{i} \in E=\mathbb{C}^{d}$ and $h \in H, L_{i}\left(R_{k} \otimes a\right)\left(f_{1} \otimes \cdots \otimes f_{k} \otimes\right.$ $h)=e_{i} \otimes R_{k}\left(f_{1} \otimes \cdots \otimes f_{k}\right) \otimes a h=\left(I_{E} \otimes R_{k}\right)\left(e_{i} \otimes f_{1} \otimes \cdots \otimes f_{k}\right) \otimes a h=$ $\left(R_{k+1} Z_{k+1} \otimes a\right) L_{i}\left(f_{1} \otimes \cdots \otimes f_{k} \otimes h\right)$. Thus

$$
L_{i}\left(R_{k} \otimes a\right)=\left(R_{k+1} Z_{k+1} \otimes a\right) L_{i}
$$

and therefore

$$
\begin{gathered}
S_{i}(T) K_{c}^{R}(T, V)\left(b a^{*}\right) S_{i}(V)^{*} \\
=\Sigma_{k=0}^{\infty} T^{(k+1)}\left(R_{k+1} Z_{k+1} \otimes b\right) L_{i} L_{i}^{*}\left(Z_{k+1}^{*} R_{k+1} \otimes a^{*}\right) V^{(k+1) *}
\end{gathered}
$$

Now fix $V_{1}, \ldots, V_{n}$ in $D_{c}(X, H)$ and $a_{1}, \ldots, a_{n}$ in $B(H)$ and consider the $n \times n$ matrix

$$
\left(S_{i}\left(V_{l}\right) K_{c}^{R}\left(V_{l}, V_{m}\right)\left(a_{l} a_{m}^{*}\right) S_{i}\left(V_{m}\right)^{*}\right)_{l, m}
$$

which, by (201), is equal to the matrix

$$
\begin{gathered}
\left(\sum_{k=0}^{\infty} V_{l}^{(k+1)}\left(R_{k+1} Z_{k+1} \otimes a_{l}\right) L_{i} L_{i}^{*}\left(Z_{k+1}^{*} R_{k+1} \otimes a_{m}^{*}\right) V_{m}^{(k+1) *}\right)_{l, m} \\
\leq\left(\sum_{k=0}^{\infty} V_{l}^{(k+1)}\left(R_{k+1} Z_{k+1} Z_{k+1}^{*} R_{k+1} \otimes a_{l} a_{m}^{*}\right) V_{m}^{(k+1) *}\right)_{l, m} \\
\leq \sup _{k}\left\|Z_{k}\right\|^{2}\left(\sum_{k=0}^{\infty} V_{l}^{(k+1)}\left(R_{k+1}^{2} \otimes a_{l} a_{m}^{*}\right) V_{m}^{(k+1) *}\right)_{l, m} \\
\leq \sup _{k}\left\|Z_{k}\right\|^{2}\left(K_{c}^{R}\left(V_{l}, V_{m}\right)\left(a_{l} a_{m}^{*}\right)\right)_{l, m}
\end{gathered}
$$

proving that $S_{i}$ is a multiplier. In fact, we see that $\left\|M_{S_{i}}\right\| \leq \sup \left\|Z_{k}\right\|(<\infty)$. This proves (1).

For (2), we define $\Lambda$ on generators by

$$
\Lambda\left(k_{a, T}^{R} \cdot c \otimes h\right)=\Sigma_{k=0}^{\infty} \oplus\left(R_{k} \otimes a^{*}\right) T^{(k) *} c h
$$

for $a, c \in B(H)$ and $T \in D_{c}(X, H)$. By Lemma 3.2, $\left(R_{k} \otimes a^{*}\right) T^{(k) *} c h \in \mathcal{R}_{k} \subseteq$ $\mathcal{F}(\mathcal{R}) \otimes H$. We now compute, for $a, b, c, d \in B(H), T, L \in D_{c}(X, H)$ and $h, g \in H$,

$$
\sum_{k=0}^{\infty}\left\langle\left(R_{k} \otimes a^{*}\right) T^{(k) *} c h,\left(R_{k} \otimes b^{*}\right) L^{(k) *} d g\right\rangle=\Sigma_{k}\left\langle h, c^{*} T^{(k)}\left(R_{k}^{2} \otimes a b^{*}\right) L^{(k) *} d g\right\rangle
$$




$$
=\left\langle h, c^{*} K^{R}(T, L)\left(a b^{*}\right) d g\right\rangle .
$$

This shows that the series defining $\Lambda\left(k_{a, T} \cdot c \otimes h\right)$ converges and, thus, belongs to $\Sigma_{k} \oplus \mathcal{R}_{k}=\mathcal{F}(\mathcal{R}) \otimes H$. We also get

$$
\begin{gathered}
\left\langle\Lambda\left(k_{a, T}^{R} \cdot c \otimes h\right), \Lambda\left(k_{b, L}^{R} \cdot d \otimes g\right)\right\rangle=\left\langle h, c^{*} K^{R}(T, L)\left(a b^{*}\right) d g\right\rangle \\
=\left\langle h,\left\langle k_{a, T}^{R} \cdot c, k_{b, L}^{R} \cdot d\right\rangle g\right\rangle=\left\langle k_{a, T}^{R} \cdot c \otimes h, k_{b, L}^{R} \cdot d \otimes g\right\rangle .
\end{gathered}
$$

Thus, $\Lambda$ is a well defined isometry into $\mathcal{F}(\mathcal{R}) \otimes H$. To show that this map is surjective, note first that, for $\lambda \in \mathbb{T}$,

$$
\Lambda\left(k_{a, \bar{\lambda} T}^{R} \cdot c \otimes h\right)=\Sigma_{k=0}^{\infty} \oplus \lambda^{k}\left(R_{k} \otimes a^{*}\right) T^{(k) *} c h
$$

We now define, for every $\lambda \in \mathbb{T}, W_{\lambda} \in B(\mathcal{F}(\mathcal{R}) \otimes H)=B\left(\Sigma_{k} \oplus \mathcal{R}_{k}\right)$ by $W_{\lambda}=\Sigma_{k} \lambda^{k} Q_{k}$ (where $Q_{k}$ is the projection onto $\mathcal{R}_{k}$ ). Then $\left\{W_{\lambda}\right\}$ is a strongly continuous group of unitaries and (21) shows that the range of $\Lambda$ is invariant under each $W_{\lambda}$. Since, for every $k \geq 0, Q_{k}=\int W_{\lambda} \lambda^{-k} d \lambda$ (in the strong operator topology), we see that the range of $\Lambda$ (which is a closed subspace) is invariant under $Q_{k}$. Since $\Lambda\left(k_{a, T}^{R} \cdot c \otimes h\right)=\Sigma_{k=0}^{\infty} \oplus\left(R_{k} \otimes a^{*}\right) T^{(k) *} c h$ for $a, c \in B(H)$ and $T \in D_{c}(X, H)$, we find that, for every $k \geq 0$ and every $a, c, T$ as above, $\left(R_{k} \otimes a^{*}\right) T^{(k) *} c h$ is in the range of $\Lambda$. It follows from Lemma 3.2 that the range of $\Lambda$ contains $\mathcal{R}_{k}$, for every $k$, and, thus, $\Lambda$ is surjective.

To prove (17) we compute.

$$
\begin{gathered}
\Lambda\left(M_{S_{i}}^{*} \otimes I_{H}\right) \Lambda^{*}\left(\sum_{k=0}^{\infty}\left(R_{k} \otimes a^{*}\right) T^{(k) *} c h\right)= \\
\Lambda\left(M_{S_{i}}^{*} \otimes I_{H}\right)\left(k_{a, T} \cdot c \otimes h\right)=\Lambda\left(k_{a, T} \cdot T_{i}^{*} c \otimes h\right) \\
=\sum_{k=0}^{\infty}\left(R_{k} \otimes a^{*}\right) T^{(k) *} T_{i}^{*} c h=\Sigma_{k=0}^{\infty}\left(R_{k} \otimes a^{*}\right) L_{i}^{*} T^{(k+1) *} c h \\
=\Sigma_{k=0}^{\infty} L_{i}^{*}\left(Z_{k+1}^{*} R_{k+1} \otimes a^{*}\right) T^{(k+1) *} c h= \\
\left(W_{i}^{*} \otimes I_{H}\right) \sum_{k=0}^{\infty}\left(R_{k+1} \otimes a^{*}\right) T^{(k+1) *} c h=\left(W_{i}^{*} \otimes I_{H}\right) \Sigma_{k=0}^{\infty}\left(R_{k} \otimes a^{*}\right) T^{(k) *} c h
\end{gathered}
$$

where we used (18), (19) and, in the last equality, the fact that $W_{i}^{*} \otimes I_{H}$ vanishes on $H$. This proves (17) and completes the proof of part (2a.).

To prove (2b.) we use the definition of $\Lambda$ and (15) and compute

$$
\begin{gathered}
\Lambda\left(\varphi_{E_{K_{c}^{R}}}(b) \otimes I_{H}\right)\left(k_{a, T}^{R} \cdot c \otimes h\right)=\Lambda\left(k_{a b^{*}, T}^{R} \cdot c \otimes h\right)=\Sigma_{k=0}^{\infty} \oplus\left(R_{k} \otimes b a^{*}\right) T^{(k) *} c h \\
=\left(I_{\mathcal{F}(\mathcal{R})} \otimes b\right) \Sigma_{k=0}^{\infty} \oplus\left(R_{k} \otimes a^{*}\right) T^{(k) *} c h=\left(I_{\mathcal{F}(\mathcal{R})} \otimes b\right) \Lambda\left(k_{a, T}^{R} \cdot c \otimes h\right) .
\end{gathered}
$$


To prove part (3) we have to show that

$$
\sum_{k=1}^{\infty} M_{S}^{(k)}\left(X_{k} \otimes I_{E_{K_{c}^{R}}}\right) M_{S}^{(k) *} \leq I .
$$

But, using part (2), it suffices to prove that

$$
\Sigma_{k=1}^{\infty} W^{(k)}\left(X_{k} \otimes I_{\mathcal{F}(\mathcal{R})}\right) W^{(k) *} \leq I
$$

and this follows from Lemma 3.4.

For the next result, recall the notion of $\sigma$-dual of a correspondence. Let $F$ be a $W^{*}$-correspondence over a $W^{*}$-algebra $M$ and let $\sigma$ be a normal representation on a Hilbert space $H$. The $\sigma$-dual, $F^{\sigma}$ is a $W^{*}$-correspondence over the $W^{*}$-algebra $\sigma(M)^{\prime}$ defined by

$$
F^{\sigma}=\left\{Y: H \rightarrow F \otimes_{\sigma} H: Y \sigma(b)=\left(\varphi_{F}(b) \otimes I_{H}\right) Y, b \in M\right\} .
$$

(See [8] for the details). The $\sigma(M)^{\prime}$-valued inner product is defined by $\left\langle Y_{1}, Y_{2}\right\rangle=Y_{1}^{*} Y_{2}$ and the bimodule structure is defined by

$$
a \cdot Y \cdot b=\left(I_{F} \otimes a\right) \circ Y \circ b
$$

for $a, b \in \sigma(M)^{\prime}$.

Proposition 5.6 Let $E_{K_{c}^{R}}$ be the correspondence over $B(H)$ associated with the kernel $K_{c}^{R}$ and $\sigma$ be the natural representation of $B(H)$ on $H$. Then

$$
E_{K_{c}^{R}}^{\sigma} \cong \mathcal{F}(\mathcal{R})
$$

where the isomorphism is an isomorphism of Hilbert spaces.

Proof. We define the map $\Psi: \mathcal{F}(\mathcal{R}) \rightarrow E_{K_{c}^{R}}^{\sigma}$ by

$$
\Psi(\xi)=\Lambda^{-1} \circ L_{\xi} \quad, \xi \in \mathcal{F}(\mathcal{R})
$$

where $L_{\xi}: H \rightarrow \mathcal{F}(\mathcal{R}) \otimes H$ is defined by $L_{\xi} h=\xi \otimes h \in \mathcal{F}(\mathcal{R}) \otimes H$ and $\Lambda$ is the map of Proposition 5.5 .

To prove that $\Psi(\xi)$ (for $\xi \in \mathcal{F}(\mathcal{R})$ ) lies in $E_{K_{c}^{R}}^{\sigma}$, fix $h \in H$ and $b \in B(H)$ and compute

$$
\Psi(\xi) \sigma(b) h=\Lambda^{-1} \circ L_{\xi} b h=\Lambda^{-1}(\xi \otimes b h)=\Lambda^{-1}\left(I_{\mathcal{F}(\mathcal{R})} \otimes b\right)(\xi \otimes h)
$$


and, using Proposition 5.5(2b.), this is equal to

$$
\left(\varphi_{E_{K_{c}^{R}}}(b) \otimes I_{H}\right) \Lambda^{-1}(\xi \otimes h)=\left(\varphi_{E_{K_{c}^{R}}}(b) \otimes I_{H}\right) \Psi(\xi) h
$$

proving that $\Psi(\xi) \in E_{K_{c}^{R} \text {. }}^{\sigma}$.

Linearity of $\Psi$ is obvious.

To show that $\Psi$ is surjective, fix $Y \in E_{K_{c}^{R}}^{\sigma}$ and write $\eta=\Lambda \circ Y: H \rightarrow$ $\mathcal{F}(\mathcal{R}) \otimes H$. For $h \in H$ and $b \in B(H)$ we get (using Proposition 5.5 (2b.)) that $\eta b h=(\Lambda \circ Y) b h=\Lambda\left(\varphi_{E_{K_{c}^{R}}}(b) \otimes I_{H}\right) Y h=\left(I_{\mathcal{F}(\mathcal{R})} \otimes b\right) \eta h$. Thus, for $b \in B(H)$,

$$
\eta b h=\left(I_{\mathcal{F}(\mathcal{R})} \otimes b\right) \eta h .
$$

Now fix a unit vector $h_{0} \in H$ and write $\eta h_{0}=\Sigma_{i} \xi_{i} \otimes h_{i}$ (for $\xi_{i} \in \mathcal{F}(\mathcal{R})$ and $\left.h_{i} \in H\right)$. Write $p_{0}$ for the projection onto $\mathbb{C} h_{0}$ and use (22) to get $\eta h_{0}=\eta p_{0} h_{0}=\Sigma_{i} \xi_{i} \otimes p_{0} h_{i}$. Thus, we can write $\eta h_{0}=\xi \otimes h_{0}$ for some $\xi \in \mathcal{F}(\mathcal{R})$. Now apply (22) again, with arbitrary $h \in H$ and $v$ which is the rank one operator mapping $h_{0}$ to $h$, to get

$$
\eta h=\eta v h_{0}=\left(I_{\mathcal{F}(\mathcal{R})} \otimes v\right) \eta h_{0}=\xi \otimes v h_{0}=\xi \otimes h
$$

proving that $\eta=L_{\xi}$ and $\Psi(\xi)=Y$.

To complete the proof we need to show that $\Psi$ is an isometry and this follows from the fact that $\Lambda^{-1}$ is an isometry and from $\left\langle L_{\xi_{1}}, L_{\xi_{2}}\right\rangle=L_{\xi_{1}}^{*} L_{\xi_{2}}=$ $\left\langle\xi_{1}, \xi_{2}\right\rangle$.

The following two theorems present our version of the Beurling-LaxHalmos Theorem for reproducing kernel correspondences.

Theorem 5.7 Suppose $E_{K}$ is an $(X, H)$-contractive reproducing kernel correspondence, $G$ is a Hilbert space and $\mathcal{S} \subseteq E_{K} \otimes_{B(H)} H \otimes G$ is a subspace. Then

(1) $\mathcal{S}$ is invariant under $M_{S} \otimes I_{H} \otimes I_{G}$ if and only if there is a Hilbert space $\mathcal{D}$ and a partial isometry $\Pi: \mathcal{F}(\mathcal{R}) \otimes \mathcal{D} \rightarrow E_{K} \otimes_{B(H)} H \otimes G$ such that, for every $i$,

$$
\left(M_{S_{i}} \otimes I_{H \otimes G}\right) \Pi=\Pi\left(W_{i} \otimes I_{\mathcal{D}}\right)
$$

and

$$
\mathcal{S}=\Pi(\mathcal{F}(\mathcal{R}) \otimes \mathcal{D})
$$


(2) Write $\mathcal{D}_{0}=H \otimes \mathcal{D}$ and let $B(H)$ act on $\mathcal{D}$ in the obvious way. Then $\mathcal{S}$ is invariant under $M_{S} \otimes I_{H} \otimes I_{G}$ if and only if there is a partial isometry $Y: E_{K_{c}^{R}} \otimes_{B(H)} \mathcal{D}_{0} \rightarrow E_{K} \otimes_{B(H)} H \otimes G$ such that, for every $i$,

$$
\left(M_{S_{i}} \otimes I_{H \otimes G}\right) Y=Y\left(M_{S_{i}} \otimes I_{\mathcal{D}_{0}}\right)
$$

and

$$
\mathcal{S}=Y\left(E_{K_{c}^{R}} \otimes_{B(H)} \mathcal{D}_{0}\right)
$$

Proof. In both (1) and (2) the condition is clearly sufficient for $\mathcal{S}$ to be an invariant subspace. So we attend to the other direction.

For (1), since we assume that $M_{S} \otimes_{B(H)} I_{H} \in \bar{D}_{c p}\left(X, E_{K} \otimes_{B(H)} H\right)$ (and, therefore also $M_{S} \otimes_{B(H)} I_{H \otimes G} \in \bar{D}_{c p}\left(X, E_{K} \otimes_{B(H)} H \otimes G\right)$ ), we can use Theorem 4.2 ,

For (2) Write $\mathcal{D}_{0}:=H \otimes \mathcal{D}$ (for $\mathcal{D}$ of part (1)). Fix a unit vector $h_{0} \in H$ and define $\Pi_{0}: \mathcal{F}(\mathcal{R}) \otimes \mathcal{D}_{0}=\mathcal{F}(\mathcal{R}) \otimes H \otimes \mathcal{D} \rightarrow E_{K} \otimes H \otimes G$ by

$$
\Pi_{0}(\xi \otimes h \otimes d)=\left\langle h, h_{0}\right\rangle \Pi(\xi \otimes d) .
$$

Clearly $\Pi_{0}$ is a well defined partial isometry with $\mathcal{S}=\Pi_{0}\left(\mathcal{F}(\mathcal{R}) \otimes \mathcal{D}_{0}\right)$ satisfying

$$
\left(M_{S_{i}} \otimes I_{H \otimes G}\right) \Pi_{0}=\Pi_{0}\left(W_{i} \otimes I_{\mathcal{D}_{0}}\right) .
$$

Now, we set $Y=\Pi_{0}\left(\Lambda \otimes I_{\mathcal{D}}\right)$. Then $\left(M_{S_{i}} \otimes I_{H \otimes G}\right) Y=\left(M_{S_{i}} \otimes I_{H \otimes G}\right) \Pi_{0}(\Lambda \otimes$ $\left.I_{\mathcal{D}}\right)=\Pi_{0}\left(W_{i} \otimes I_{\mathcal{D}_{0}}\right)\left(\Lambda \otimes I_{\mathcal{D}}\right)=\Pi_{0}\left(\Lambda \otimes I_{\mathcal{D}}\right)\left(M_{S_{i}} \otimes I_{\mathcal{D}_{0}}\right)=Y\left(M_{S_{i}} \otimes I_{\mathcal{D}_{0}}\right)$.

Definition 5.8 Suppose $K_{1}, K_{2}$ are $\mathbb{C}$-valued positive kernels on a set $\Sigma$. Then the elements of the reproducing kernel Hilbert space $H_{K_{i}}$ are functions on $\Sigma$ and, if $G_{1}, G_{2}$ are Hilbert spaces we can view the elements of $H_{K_{i}} \otimes G_{i}$ as functions from $\Sigma$ to $G_{i}$ in a natural way. An operator-valued map $\Theta$ : $\Sigma \rightarrow B\left(G_{1}, G_{2}\right)$ is said to be a $K_{1}-K_{2}$-multiplier if, for every $f \in H_{K_{1}} \otimes G_{1}$ (viewed as a function from $\Sigma$ to $G_{1}$ ), the function $\Theta f$ lies in $H_{K_{2}} \otimes G_{2}$. In such a case, we write $M_{\Theta}$ for the map that sends $f$ to $\Theta f$ and write $\Theta \in \mathcal{M}\left(H_{K_{1}} \otimes G_{1}, H_{K_{2}} \otimes G_{2}\right)$.

A multiplier $\Theta$ is said to be partially isometric if $M_{\Theta}$ is.

Now note what we get for the case $H=\mathbb{C}$. In this case $D(X, \mathbb{C})=$ $D_{c}(X, \mathbb{C})=\left\{z=\left(z_{1}, \cdots, z_{d}\right) \in \mathbb{C}^{d}: \Sigma_{|\alpha|=|\beta|} z_{\alpha} \overline{z_{\beta}} x_{\alpha, \beta}<1\right\}$. The kernel $K_{c}^{R}$ can be viewed as a $\mathbb{C}$-valued kernel and, as we saw in Proposition 3.1, $E_{K_{c}^{R}}$ 
is the weighted Hilbert space $\mathcal{F}(\mathcal{R})=\Sigma R_{k}\left(\mathbb{C}^{d}\right)^{\circledR k}$. In this case, $K$ in the statement of the theorem is a $\mathbb{C}$-valued kernel defined on $D_{c}(X, \mathbb{C}) \times D_{c}(X, \mathbb{C})$ and giving rise to a RKHS $E_{K}$ such that

$$
\Sigma_{k=1}^{\infty} M_{z}^{(k)}\left(X_{k} \otimes I_{E_{K}}\right) M_{z}^{(k) *}=\Sigma_{|\alpha|=|\beta|} x_{\alpha, \beta} M_{z_{\alpha}} M_{z_{\beta}}^{*} \leq I .
$$

Thus, we get

Theorem 5.9 Suppose $K: D(X, \mathbb{C}) \times D(X, \mathbb{C}) \rightarrow \mathbb{C}$ is a positive definite kernel where $D(X, \mathbb{C})=\left\{z=\left(z_{1}, \cdots, z_{d}\right) \in \mathbb{C}^{d}: \Sigma_{|\alpha|=|\beta|} z_{\alpha} \overline{z_{\beta}} x_{\alpha, \beta}<1\right\}$. Also assume that $M_{z}$ is a bounded multiplier on the RKHS $E_{K}$ and it satisfies (23). Then, given a Hilbert space $G$ and a subspace $\mathcal{S} \subseteq E_{K} \otimes G$,

(1) $\mathcal{S}$ is invariant under $M_{z} \otimes I_{G}$ if and only if there is a Hilbert space $\mathcal{D}$ and a partially isometric map $\Pi: \mathcal{F}(\mathcal{R}) \otimes \mathcal{D} \rightarrow E_{K} \otimes G$ such that for every $i$,

$$
\left(M_{z_{i}} \otimes I_{G}\right) \Pi=\Pi\left(W_{i} \otimes I_{\mathcal{D}}\right)
$$

and

$$
\mathcal{S}=\Pi(\mathcal{F}(\mathcal{R}) \otimes \mathcal{D})
$$

(2) $\mathcal{S}$ is invariant under $M_{z} \otimes I_{G}$ if and only if there is a partially isometric multiplier $\Theta \in \mathcal{M}\left(E_{K_{c}^{R}} \otimes \mathcal{D}, E_{K} \otimes G\right)$ such that

$$
\mathcal{S}=M_{\Theta}\left(E_{K_{c}^{R}} \otimes \mathcal{D}\right) .
$$

(3) When $K=K_{c}^{R}$ (viewed as a $\mathbb{C}$-valued kernel), $\mathcal{S} \subseteq \mathcal{F}(\mathcal{R}) \otimes G$ is invariant under $M_{z} \otimes I_{G}$ if and only if there is a partially isometric multiplier $\Theta \in \mathcal{M}(\mathcal{F}(\mathcal{R}) \otimes \mathcal{D}, \mathcal{F}(\mathcal{R}) \otimes G)$ such that

$$
\mathcal{S}=M_{\Theta}(\mathcal{F}(\mathcal{R}) \otimes \mathcal{D})
$$

Proof. Everything follows from Theorem 5.7 except for the fact that the map $Y$ in part (2) is $M_{\Theta}$ for some multiplier $\Theta \in \mathcal{M}\left(E_{K_{c}^{R}} \otimes \mathcal{D}, E_{K} \otimes G\right)$. Applying Theorem 5.7(2), we get a partial isometry $Y: E_{K_{c}^{R}} \otimes \mathcal{D} \rightarrow E_{K} \otimes G$ such that, for every $i$,

$$
\left(M_{z_{i}} \otimes I_{G}\right) Y=Y\left(M_{z_{i}} \otimes I_{\mathcal{D}}\right)
$$

and

$$
\mathcal{S}=Y\left(E_{K_{c}^{R}} \otimes \mathcal{D}\right)
$$


To prove that $Y=M_{\Theta}$ we first follow the proof of [15, Lemma 2.2] (using also Lemma 3.5) to show that for every $w \in D(X, \mathbb{C})$ there is a map $\Theta(w) \in$ $B(\mathcal{D}, G)$ such that, for every $g \in G$,

$$
Y^{*}\left(k_{w} \otimes g\right)=k_{w}^{R} \otimes \Theta(w)^{*} g
$$

where $k_{w}, k_{w}^{R}$ are the reproducing functions of $K, K_{c}^{R}$ respectively. Write $A(z, w)=K(z, w) I_{G}-\Theta(z) K_{c}^{R}(z, w) \Theta(w)^{*} \in B(G)$ and compute,

$$
A(z, w)=\left\langle k_{z}, k_{w}\right\rangle I_{G}-\Theta(z)\left\langle k_{z}^{R}, k_{w}^{R}\right\rangle \Theta(w)^{*} .
$$

Thus, for $g, h \in G$,

$$
\begin{gathered}
\langle A(z, w) g, h\rangle=\left\langle k_{w}, k_{z}\right\rangle\langle g, h\rangle-\left\langle k_{w}^{R} \otimes \Theta(w)^{*} g, k_{z}^{R} \otimes \Theta(z)^{*} h\right\rangle= \\
\left\langle k_{w}, k_{z}\right\rangle\langle g, h\rangle-\left\langle Y^{*}\left(k_{w} \otimes g\right), Y^{*}\left(k_{z} \otimes h\right)\right\rangle=\left\langle k_{w}, k_{z}\right\rangle\langle g, h\rangle-\left\langle k_{w} \otimes g, Y Y^{*}\left(k_{z} \otimes h\right)\right\rangle= \\
\left\langle k_{w} \otimes g,\left(I-Y Y^{*}\right)\left(k_{z} \otimes h\right)\right\rangle .
\end{gathered}
$$

It follows that $A(z, w)$ is positive definite and, therefore, $\Theta$ is a multiplier.

Note that part (3) of the theorem is closely related to [12, Theorem 3.3] and to [15, Theorem 2.3].

Restricting to the case $R_{k}=I$ for all $k$ (or, equivalently, $X_{1}=I$ and $X_{k}=0$ for $k>1$ ) we get [3, Theorem 6.5].

In the analysis above, condition (16) plays an important role. It generalizes the condition that $M_{S}$ is contractive. In order to better understand condition (16) we shall first need the following lemma.

Lemma 5.10 For $T, L$ in $D_{c}(X, H)$ the map $\Phi_{T, L}: B(H) \rightarrow B(H)$ defined by

$$
\Phi_{T, L}(a)=\Sigma_{k=1}^{\infty} T^{(k)}\left(X_{k} \otimes a\right) L^{(k)}
$$

is bounded with $\left\|\Phi_{T, L}\right\|<1$ and

$$
\left(i d-\Phi_{T, L}\right)^{-1}=K_{c}^{R}(T, L) .
$$

This can be viewed as the operator version of (2).

Proof. Recall (Equation (4) ) that $\Phi_{T}(a)=\sum_{k=1}^{\infty} T^{(k)}\left(X_{k} \otimes a\right) T^{(k) *}$. This is a completely positive map on $B(H)$ and, thus, $\left\|\Phi_{T}\right\|=\left\|\Phi_{T}(I)\right\|$. 
Fix $a \in B(H)$ and consider the rows of operators $A:=\left(T^{(k)}\left(X_{k}^{1 / 2} \otimes a\right)\right)_{k=1}^{\infty}$ and $B:=\left(L^{(k)}\left(X_{k}^{1 / 2} \otimes I_{H}\right)\right)_{k=1}^{\infty}$. Then $A A^{*}=\Phi_{T}\left(a a^{*}\right), B B^{*}=\Phi_{L}(I)$ and $A B^{*}=\Phi_{T, L}(a)$. Thus

$$
\left\|\Phi_{T, L}(a)\right\| \leq\|A\|\|B\| \leq\left\|\Phi_{T}\right\|^{1 / 2}\|a\|\left\|\Phi_{L}\right\|^{1 / 2} .
$$

Since $T$ and $L$ lie in $D_{c}(X, H),\left\|\Phi_{T}\right\|<1$ and also $\left\|\Phi_{L}\right\|<1$ and it follows that $\left\|\Phi_{T, L}\right\|<1$. Therefore $\left(i d-\Phi_{T, L}\right)^{-1}$ is a well defined map on $B(H)$ that is equal to $\Sigma_{k \geq 0} \Phi_{T, L}^{k}$. A computation very much like in [9, page 516] shows that

$$
\left(i d-\Phi_{T, L}\right)^{-1}=K_{c}^{R}(T, L) .
$$

(There it is done for $T=L$ ).

Theorem 5.11 For a kernel $K: D_{c}(X, H) \times D_{c}(X, H) \rightarrow B_{*}(B(H), B(H))$ and $\Phi_{T, L}$ as in Lemma 5.10 ,

(1) Condition (16) is equivalent to the condition that $\left(i d-\Phi_{T, L}\right) \circ K(T, L)$ is a cp kernel.

(2) Condition (16) is equivalent to the condition that $\left(K_{c}^{R}(T, L)\right)^{-1} \circ K(T, L)$ is a cp kernel.

Proof. Once we prove part (1), part (2) will follow from Lemma 5.10, So it suffices to prove part (1).

$\left(i d-\Phi_{T, L}\right) \circ K(T, L)$ is a cp kernel means that, for every $T^{1}, \ldots, T^{k}, L^{1}, \ldots, L^{k}$ in $D_{c}(X, H)$ and every $a_{1}, \ldots, a_{k}$ in $B(H)$, the matrix (with entries in $B(H)$ ) defined by

$$
\left(\left(i d-\Phi_{T^{i}, L^{j}}\right) \circ K\left(T^{i}, L^{j}\right)\left(a_{i} a_{j}^{*}\right)\right)_{i, j=1}^{k}
$$

is positive. Since

$$
K\left(T^{i}, L^{j}\right)\left(a_{i} a_{j}^{*}\right)=\left\langle k_{a_{i}, T^{i}}, k_{a_{j}, L^{j}}\right\rangle
$$

we have

$$
\begin{gathered}
\Phi_{T^{i}, L^{j}}\left(K\left(T^{i}, L^{j}\right)\left(a_{i} a_{j}^{*}\right)\right)=\Sigma_{|\alpha|=|\beta|} x_{\alpha, \beta} T_{\alpha}^{i}\left\langle k_{a_{i}, T^{i}}, k_{a_{j}, L^{j}}\right\rangle L_{\beta}^{j *}= \\
\Sigma x_{\alpha, \beta}\left\langle k_{a_{i}, T^{i}} \cdot T_{\alpha}^{i *}, k_{a_{j}, L^{j}} \cdot L_{\beta}^{j *}\right\rangle=\Sigma x_{\alpha, \beta}\left\langle M_{S_{\alpha}}^{*} k_{a_{i}, T^{i}}, M_{S_{\beta}}^{*} k_{a_{j}, L^{j}}\right\rangle= \\
\left\langle k_{a_{i}, T^{i}}, \Sigma x_{\alpha, \beta} M_{S_{\alpha}} M_{S_{\beta}}^{*} k_{a_{j}, L^{j}}\right\rangle
\end{gathered}
$$


and

$$
\left(i d-\Phi_{T^{i}, L^{j}}\right) \circ K\left(T^{i}, L^{j}\right)\left(a_{i} a_{j}^{*}\right)=\left\langle k_{a_{i}, T^{i}},\left(I-\Sigma x_{\alpha, \beta} M_{S_{\alpha}} M_{S_{\beta}}^{*}\right) k_{a_{j}, L^{j}}\right\rangle .
$$

Thus, the positivity of $\left(i d-\Phi_{T, L}\right) \circ K(T, L)$ is equivalent to $I-\Sigma x_{\alpha, \beta} M_{S_{\alpha}} M_{S_{\beta}}^{*} \geq$ 0 which is (16).

Example 5.12 Suppose $\left\{B_{k}^{2}\right\}_{k=0}^{\infty}$ is a sequence of positive operators, $B_{k} \in$ $B\left(\left(\mathbb{C}^{d}\right)^{\otimes k}\right)$, and the kernel $K^{B}(T, L)(a):=\sum_{k=0}^{\infty} T^{(k)}\left(B_{k}^{2} \otimes a\right) L^{(k) *}$ is well defined on $D_{c}(X, H)$ (where $a \in B(H)$ ). Define

$$
C_{k}^{2}=\sum_{m=0}^{k} R_{m}^{2} \otimes B_{k-m}^{2} \in B\left(\left(\mathbb{C}^{d}\right)^{\otimes k}\right)
$$

and write

$$
K^{C}(T, L)(a):=\Sigma_{k=0}^{\infty} T^{(k)}\left(C_{k}^{2} \otimes a\right) L^{(k) *} .
$$

Then a straightforward computation shows that

$$
K^{C}(T, L)=K_{c}^{R}(T, L) \circ K^{B}(T, L) .
$$

Thus, using Theorem 5.11 (2), $K^{C}$ satisfies condition (16) and $E_{K}$ is an $(X, H)$-contractive reproducing kernel correspondence.

In particular, this holds when $B_{k}=R_{k}$ so that

$$
C_{k}^{2}=\sum_{m=0}^{\infty} R_{m}^{2} \otimes R_{k-m}^{2}
$$

For example, if $R_{k}=I$ for all $k$, we get $C_{k}^{2}=(k+1) I$ and $E_{K^{C}}$ can be viewed as a generalization of the Bergman space.

\section{References}

[1] J. Ball and V. Bolotnikov, A Beurling type theorm in weighted Bergman spaces C. R. Math. Acad. Sci. Paris 351 (2013), 433-436.

[2] J. Ball and V. Bolotnikov, Weighted Bergman spaces: shift-invariant spaces and input/state/output linear systems Int. Eq. Oper. Theory 76 (2013), 301-356.

[3] M. Bhattacharjee, J. Eschmeier, D. K. Keshari and J. Sarkar, Dilations, wandering subspaces and inner functions LAAA 523 (2017), 263-280. 
[4] R. Clouâtre, M. Hartz and D. Schillo, A Beurling-Lax-Halmos theorem for spaces with a complete Nevanlinna-Pick factor Proc. Amer. Math. Soc. 148 (2020), 731-740.

[5] J. Good, Weighted interpolation in $W^{*}$-algebras Ph.D. Thesis, University of Iowa (2015).

[6] S. McCullough and T. Trent, Invariant subspaces and Nevanlinna-Pick kernels J. Funct. Anal. 178 (2000), 226-249.

[7] J. Meyer, Noncommutative Hardy algebras, multipliers and quotients Ph.D. Thesis, University of Iowa (2010).

[8] P. Muhly and B. Solel, Hardy algebras, $W^{*}$-correspondences and interpolation theory Math. Ann. 330 (2) (2004), 353-415.

[9] P. Muhly and B. Solel, Matricial function theory and weighted shifts Int. Eq. Op. Thy. 84 (2016), 501-553.

[10] P. Muhly and B. Solel, The Poisson kernel for Hardy algebras Compl. Anal. Oper. Theory 3 (2009), 221-242.

[11] G. Popescu, Poisson transforms on some $C^{*}$-algebras generated by isometries J. Funct. Anal. 161, (1999), 27-61.

[12] G. Popescu, Operator theory on noncommutative domains Mem. Amer. Math. Soc. 205 (964), (2010), vi+124.

[13] V. Paulsen and M. Raghupathi, An introduction to the theory of reproducing kernel Hilbert spaces

[14] H. Radjavi and P. Rosenthal, Invariant subspaces, Springer-Verlag New York Heidelberg Berlin 1973.

[15] J. Sarkar, An invariant subspace theorem and invariant subspaces of analytic reproducing kernel Hilbert spaces. I J. Operator Theory 73 (2015), 433-441.

[16] J. Sarkar, An invariant subspace theorem and invariant subspaces of analytic reproducing kernel Hilbert spaces II Complex Anal. Oper. Theory 10 (2016), 769-782. 